\title{
Abilities of berberine and chemically modified berberines to interact with metformin and inhibit proliferation of pancreatic cancer cells
}

\author{
Shaw M. Akula ${ }^{\mathrm{a}, *, 1}$, Saverio Candido ${ }^{\mathrm{b}, \mathrm{c}, 1}$, Massimo Libra ${ }^{\mathrm{b}, \mathrm{c}}$, Stephen L. Abrams ${ }^{\mathrm{a}}$, \\ Linda S. Steelman $^{\text {a }}$, Kvin Lertpiriyapong ${ }^{\mathrm{d}}$, Giulia Ramazzotti ${ }^{\mathrm{e}}$, Stefano Ratti ${ }^{\mathrm{e}}$, \\ Matilde Y. Follo ${ }^{\mathrm{e}}$, Alberto M. Martelli ${ }^{\mathrm{e}}$, Ramiro M. Murata, ${ }^{\mathrm{a}, \mathrm{f}}$, Pedro L. Rosalen ${ }^{\mathrm{g}}$, \\ Bruno Bueno-Silva ${ }^{\text {g,h }}$, Severino Matias de Alencar ${ }^{\mathrm{i}}$, Giuseppe Montalto ${ }^{\mathrm{j}, \mathrm{k}}$, \\ Melchiorre Cervello $^{\mathrm{k}}$, Agnieszka Gizak ${ }^{1}$, Dariusz Rakus ${ }^{1}$, Weifeng Mao ${ }^{\mathrm{m}}$, \\ Heng-Liang Lin ${ }^{\mathrm{n}}$, Paolo Lombardi ${ }^{\circ}$, James A. McCubrey ${ }^{\mathrm{a}, * *}$ \\ ${ }^{a}$ Department of Microbiology and Immunology, Brody School of Medicine at East Carolina University, Greenville, NC, 27858, USA \\ ${ }^{\mathrm{b}}$ Department of Biomedical and Biotechnological Sciences - Oncological, Clinical and General Pathology Section, University of Catania, Catania, Italy \\ ${ }^{c}$ Research Center for Prevention, Diagnosis and Treatment of Cancer (PreDiCT), University of Catania, Catania, Italy \\ ${ }^{\mathrm{d}}$ Center of Comparative Medicine and Pathology, Memorial Sloan-Kettering Cancer Center, Weill Cornell Medicine and the Hospital for Special \\ Surgery, New York City, New York, USA \\ e Dipartimento di Scienze Biomediche e Neuromotorie, Università di Bologna, Bologna, Italy \\ ${ }_{\mathrm{f}}^{\mathrm{f}}$ Department of Foundational Sciences, School of Dental Medicine, East Carolina University, USA \\ ${ }^{g}$ Department of Physiological Sciences, Piracicaba Dental School, State University of Campinas, Piracicaba, Brazil \\ ${ }^{\mathrm{h}}$ Dental Research Division, Guarulhos University, Guarulhos, Brazil \\ i Universidade de São Paulo - USP, "Luiz de Queiroz" College of Agriculture, São Paulo, Brazil \\ ${ }^{\mathrm{j}}$ Dipartimento di Promozione Della Salute, Materno-Infantile, Medicina Interna e Specialistica di Eccellenza (PROMISE), University of Palermo, \\ Palermo, Italy \\ ${ }^{\mathrm{k}}$ Consiglio Nazionale Delle Ricerche, Istituto di Biomedicina e Immunologia Molecolare "Alberto Monroy", Palermo, Italy \\ ${ }^{1}$ Department of Molecular Physiology and Neurobiology, Wroclaw University, Wroclaw, Poland \\ ${ }^{\mathrm{m}}$ College of Basic Medical Sciences, Dalian Medical University, Dalian, Liaoning, China \\ ${ }^{\mathrm{n}}$ Catholic Fu Jen University Hospital, New Taipei City, Taiwan \\ ${ }^{\circ}$ Naxospharma, Via Giuseppe di Vittorio 70, Novate Milanese, 20026, Italy
}

\section{A R T I C L E I N F O}

\section{Keywords:}

Berberine

TP53

PDAC

Signal transduction inhibitors

\begin{abstract}
A B S T R A C T
Pancreatic cancer is devastating cancer worldwide with few if any truly effective therapies. Pancreatic cancer has an increasing incidence and may become the second leading cause of death from cancer. Novel, more effective therapeutic approaches are needed as pancreatic cancer patients usually survive for less than a year after being diagnosed. Control of blood sugar levels by the prescription drug metformin in diseases such as diabetes mellitus has been examined in association with pancreatic cancer. While the clinical trials remain inconclusive, there is hope that certain diets and medications may affect positively the outcomes of patients with pancreatic and other cancers. Other natural compounds may share some of the effects of metformin. One "medicinal" fruit consumed by millions worldwide is berberine (BBR). Metformin and BBR both activate AMP-activated protein kinase (AMPK) which is a key mediator of glucose metabolism.
\end{abstract}

\footnotetext{
* Corresponding author.

** Corresponding author.

E-mail addresses: akulas@ecu.edu (S.M. Akula), mccubreyj@ecu.edu (J.A. McCubrey).

${ }^{1}$ Co-first authors: Shaw M. Akula and Saverio Candido. These authors contributed equally to these studies.
} 


\begin{abstract}
Glucose metabolism has been shown to be very important in cancer and its significance is increasing. In the following studies, we have examined the effects of metformin, BBR and a panel of modified BBRs (NAX compounds) and chemotherapeutic drugs on the growth of four different human pancreatic adenocarcinoma cell lines (PDAC). Interestingly, the effects of metformin could be enhanced by BBR and certain modified BBRs. Upon restoration of WT-TP53 activity in MIA-PaCa-2 cells, an altered sensitivity to the combination of certain NAX compounds and metformin was observed compared to the parental cells which normally lack WT-TP53. Certain NAX compounds may interact with WT-TP53 and metformin treatment to alter the expression of key molecules involved in cell growth. These results suggest a therapeutic approach by combining certain pharmaceutical drugs and nutraceuticals to suppress the growth of cancer cells.
\end{abstract}

\title{
1. Introduction
}

Previously, we have summarized many of the key genetic and biological factors implicated in pancreatic cancer (Candido et al., 2018; Abrams et al., 2018, 2019; McCubrey et al., 2018). Although various genetics and environmental factors may be involved in pancreatic cancer, few remedies have been developed to treat this devastating disease beyond surgery and chemotherapy which, unfortunately, only have minimal positive effects on patient survival. Clearly novel, more effective approaches are needed to treat pancreatic ductal adenocarcinoma (PDAC) and the subsequent drug resistance which may arise.

Metformin (N,N-dimethylimidodicarbonimidic diamide) is a type II diabetes drug which also has effects on cancer (Lonardo et al., 2013; Candido et al., 2018). Metformin mainly blocks hepatic gluconeogenesis. Cancer cells normally have high glucose requirements due to their frequent utilization of the inefficient breakdown of glucose by the anerobic pyruvate pathway (Warburg effect) to generate ATP. Thus, metformin can have anti-cancer effects by modulation of glucose and energy levels in cancer cells. Epidemiological studies have suggested that metformin may have effects on PDAC development.

However, the relationship between metformin use and PDAC development and survival remains controversial (Bosetti et al., 2014). While some studies suggested a benefit for PDAC patients who consumed metformin (Cerullo et al., 2017) other investigations

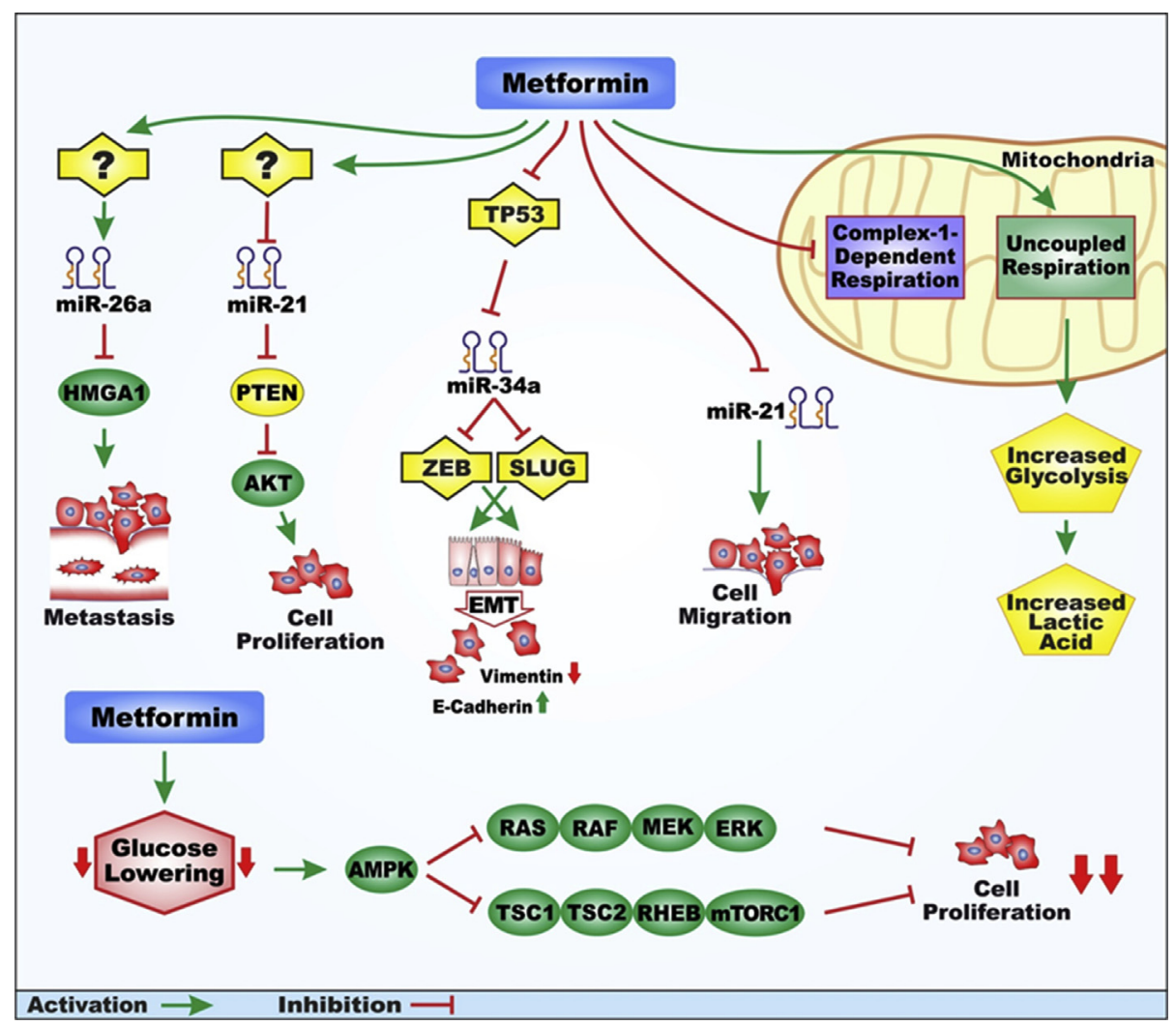

Fig. 1. Effects of Metformin on Metabolism, Gene Expression, Induction of miRs, Cellular Proliferation and Metastasis. Metformin treatment results in the modulation of various metabolic pathways which play key roles in the regulation of cellular proliferation. Various miRs are induced by metformin. This figure depicts briefly, how these miR may affect certain signal transduction and apoptotic pathways which are important in the regulation of cellular growth, malignant transformation, invasion, and drug resistance. 
did not demonstrate a statistically significant difference in survival levels (Chaiteerakij et al., 2016). Moreover, in a study comparing metformin consumption to a standard systemic therapy (e.g., cisplatin, epirubicin, capecitabine and gemcitabine), a significant increase in outcome of patients with metastatic pancreatic cancer was not observed (Reni et al., 2016). Data from 907 patients in the Netherlands Comprehensive Cancer Center observational cohort (1998-2011) indicated that there was no association in terms of survival in patients using metformin in PDAC patients (Frouws et al., 2017). However, there are at least 16 clinical trials with the anti-type II diabetes drug metformin and pancreatic cancer [https: $/ /$ clinicaltrials.gov $/ \mathrm{ct} 2 /$ results? cond $=$ pancreatic + cancer\&term $=$ metformin $\&$ cntry $=\&$ state $=\&$ city $=\&$ dist $=$ ]. Thus, the association between metformin use and PDAC is controversial (Chaiteerakij et al., 2016). However, it should be emphasized that metformin has been proposed to have effects on cancer stem cells (CSC) and sensitivity to chemotherapy (Hirsch et al., 2009, 2013; Iliopoulos et al., 2011; Rattan et al., 2011; Bao et al., 2012, 2014; Xu et al., 2018).

\subsection{Regulation of key signaling pathways by metformin}

Metformin activates $5^{\prime}$ AMPK and suppresses mTORC1 activity, a key regulator of cell growth and the target of the immunosuppressive, anti-cancer drug rapamycin (Ming et al., 2014). When AMPK is activated, the pro-proliferative mTORC1 and RAF/ MEK/ERK pathways are inhibited. These pathways function to regulate many processes involved in cell growth, the prevention of apoptosis and drug resistance. The PI3K/PTEN/Akt/mTORC1 and RAF/MEK/ERK pathways often serve to regulate various growth regulatory mRNAs which have 3'regions structures which make them difficult to translate by phosphorylation of key proteins involved with ribosomes and effect protein translation.

\subsection{Regulation of microRNAs (miRs) by metformin}

Some of the key molecules involved in drug resistance affected by metformin treatment are miRs. miRs regulate expression of genes. miRs are small non-coding RNAs (ncRNA), 19-25 nucleotides in length, derived from hairpin-shaped precursor molecules encoded by the genomes of animals, plants, and viruses (Esteller, 2011). miRNAs control gene expression and regulate a wide array of biological processes by targeting mRNAs and inducing translational repression or RNA degradation (Adams et al., 2014). Among mammals, miR coding sequences are estimated to account for about $1 \%$ of the genome, and yet more than $60 \%$ of protein coding genes are regulated by miRs (Friedman et al., 2009). A diagram of the effects of metformin on some miRs and cellular growth and metastasis is presented in Fig. 1. The effects of metformin on miRs is currently under intense study. Some studies have indicated that miRs may be induced by metformin. In a hyperglycemia model consisting of mouse microvascular endothelial cells (MMEC), miR-34a expression altered sirtuin 1 expression as well as post-translation modification of endothelial nitric oxide synthase and angiogenesis. Metformin treatment or inhibition of miR-34a, increased expression of sirtuins and prevented the impairment of angiogenesis. Overexpression of a miR-34a mimic was determined to suppress the effects of metformin (Arunachalam et al., 2016).

Metformin can reduce glucose levels in PDAC cells. This resulted in decreased levels of miR-34a and its downstream targets NOTCH, SLUG and SNAIL. The frequency of PDAC spheroid formations as well as the spheroid sizes were reduced (Cifarelli et al., 2015).

Metformin was also demonstrated to increase the expression of certain miRs in PDAC cells including: let-7a, let-7b, miR-26a, miR110, miR-200b and miR-200c (Bao et al., 2012). These resulted in the decreased expression of CD44, epithelial cell adhesion molecule (EpCAM), enhancer of zeste homolog 2 (EZH2), NOTCH-1, NANOG and OCT4. Re-expression of miR-26a, mediated by transfection, resulted in decreased expression of EZH2 and EpCAM.

Other studies have shown that metformin can induce miR-34a, which results in the down-regulation of SIRT1/PGC1 $\alpha /$ NRF2 pathway in cells with wild type (WT) TP53 (Do et al., 2014). Metformin resulted in decreased levels of SIRT1 in cells with WT-TP53 but not in cells with mutant TP53. In cells with WT-TP53, metformin induced TP53 and miR-34a and suppressed peroxisome proliferator activated receptor $\delta$ (PPAR $\delta$ ) coactivator $1 \alpha$ (PGC-1 $\alpha$ ). This resulted in the suppression of nuclear factor-like 2 (NRF2), at least in TP53-positive MCF-7 breast cancer cells (Do et al., 2014).

Metformin inhibits certain miRs in rat mesangial cells cultured with high glucose (Wu et al., 2018) High glucose treatment normally results in activation of miRs and the early growth response protein 1 (EGR1) transcription factor. miR-34 can suppress SIRT1 and AMPK which results in EGR1 activation. Metformin suppressed this induction.

Metformin was shown to inhibit the proliferation, migration and invasion in HCT116 and SW-480 colorectal cancer (CRC) cells (Wang et al., 2018). Metformin treatment resulted in the up-regulation of E-cadherin and downregulation of vimentin during the process of epithelial to mesenchymal transition (EMT) of CRC cells. In these studies, metformin induced miR-200a, miR-200c and miR-429 but decreased miR-34a. Metformin treatment also resulted in decreased levels of Snail and ZEB expression.

Metformin also suppressed miR-27a expression in PDAC cells which resulted in increased expression of zinc finger and BR-C, ttk and bab (BTB) domain containing 10 (ZBTB10). ZBTB10 is a suppressor of the SP1, SP3 and SP4 transcription factors. Metformin treatment resulted in a decreased growth of the PDAC cells both in vitro and in orthotopic tumor models (Nair et al., 2013).

\subsection{Regulation of signal transduction pathways, cellular growth, and apoptosis by berberine}

Berberine (BBR) is a commonly-consumed nutraceutical. We have recently summarized the effects of BBR on many important processes involved in cell growth (Abrams et al., 2018a; Abrams et al., 2018b; McCubrey et al., 2017, 2018). The pleiotropic effects on various properties on the regulation of cell growth and apoptosis have also been critically evaluated recently (Farooqi et al., 2019). 
Below, we summarize some of the more novel aspects about BBRs which may explain some of their diverse effects on cellular proliferation and drug resistance. BBR can also activate AMPK (Pan et al., 2017). BBR has been shown to modulate the activity of reactive oxygen species (ROS) levels (Chatterjee et al., 2015). ROS can alter the activity of many signaling and apoptotic pathways including RAS/RAF/MEK/ERK and mTORC1 pathways (Burgeiro et al., 2011; Hsu et al., 2012; McCubrey et al., 2017, 2018). BBRs can also have effects on NF-кB, TP53 and other transcription factors which are key controllers of gene regulation, DNA repair and metastasis. A diagram summarizing the effects of BBR on gene expression, cellular migration, DNA/chromatin structure and cell cycle progression is presented in Fig. 2.

Some of the potential anti-diabetic and anti-cancer effects of BBRs are their abilities to localize to the mitochondria and inhibit the electron transport chain and activate AMPK and suppress mTOR activity (Wang et al., 2010). The PI3K/PTEN/AKT/mTORC1 and $\mathrm{RAF} / \mathrm{MEK} / \mathrm{ERK}$ pathways are inhibited when AMPK is activated.

\subsection{BBR can alter gene expression through DNA binding}

Some of the more intriguing effects of BBR and modified BBRs may be due to their interactions with nucleic acids. BBR may bind DNA and RNA via the nitrogen atom at the 7-positon in the alkaloid BBR skeleton. Different modified BBRs may have distinct interactions with nucleic acids which confer diverse effects on gene expression. Interactions between BBRs and nucleic acids may inhibit telomerases and topoisomerases as well as gene transcript levels by interacting with the TATA-binding protein and TATA-box present in certain gene promoters (Gatto et al., 1996; Qin et al., 2007; Bhowmik et al., 2012a Xiao et al., 2012). Thus, different modified BBRs might have diverse effects on gene expression and cellular growth which could be exploited commercially.

In addition, BBRs may induce double strand DNA breaks and cell cycle arrest (Wang et al., 2012). BBRs can also influence gene expression by inhibiting histone deacetylase (HDAC) activity. BBR repressed total HDAC and class I, II and IV HDAC activity by hyperacetylation of histones in A549 lung cancer cells. This resulted in decreases in prostaglandin-endoperoxide synthase 2 (COX2),

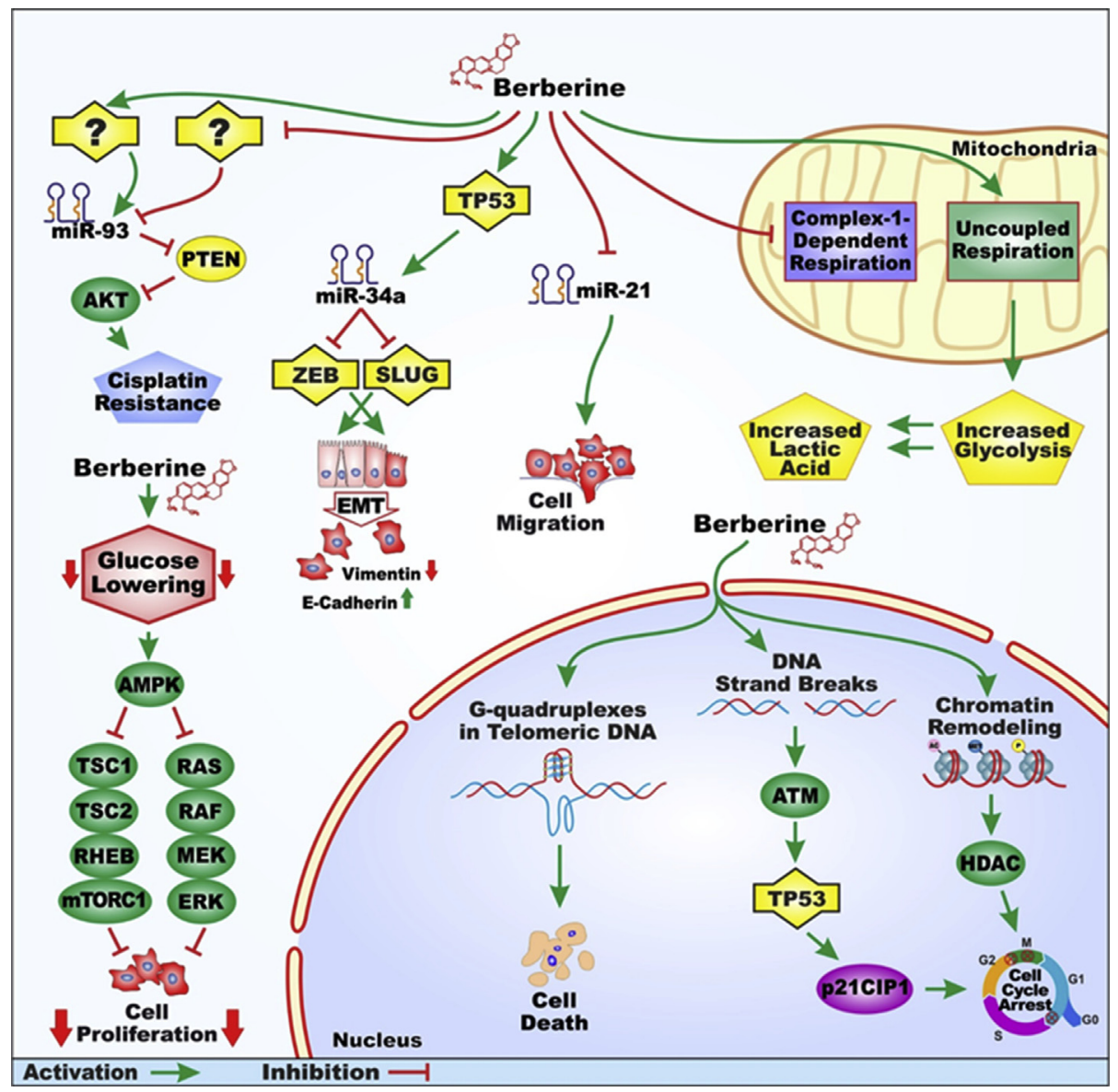

Fig. 2. Effects of Berberine on Metabolism, Gene Expression, Induction of miRs, Cellular Proliferation and Metastasis. Berberine has effects on many cellular processes including: metabolism, regulation of gene expression by interaction with DNA and chromatin in the nucleus and signaling pathways. Various miRs are induced by BBR. The mechanisms by which they may affect certain signal transduction and apoptotic pathways which are important in the regulation of cellular growth, malignant transformation, invasion, and drug resistance are depicted briefly in this figure. 
matrix metalloproteinase (MMP2), MMP9 and tumor necrosis factor-alpha (TNF- $\alpha$ ) expression and increases in TP53 and p21 Cip-1 levels. BBR was determined to regulate apoptotic activity (Kalaiarasi et al., 2016). In this system, BBR could have both direct and indirect effects on DNA by altering chromatin structure and TP53 expression. Thus, there are important interactions between BBR and TP53.

\subsection{BBR effects on cisplatin-resistance}

Cisplatin normally functions by the induction of DNA strand breaks. BBR can sensitize breast cancer cells to cisplatin and inhibit growth, however, in some cases cisplatin treatment is ineffective due to the development of drug-resistance. BBR may increase the effectiveness of cisplatin in inducing DNA strand breaks by augmenting the activation of caspase-3, caspase-9 and apoptosis (Zhao et al., 2016).

In the cisplatin-sensitive OV2008 cell line and in the resistant $\mathrm{C} 13^{*}$ subline, BBR differently affected cell growth of sensitive and resistant cells. BBR suppressed the growth of cisplatin-resistant cells more than the sensitive counterparts. This was demostrated to occur by altered expression of genes encoding folate cycle enzymes, such as dihydrofolate reductase (DHFR) and thymidylate synthase (TS). BBR also affected polyamine metabolism. This occurred by upregulation of the key catabolic enzyme, spermidine/ spermine N1-acetyltransferase (SSAT). BBR stimulated SSAT induction by the spermine analogue N1, N12 bisethyl $\neg$ spermine (BESpm). BESpm downregulated DHFR mRNA more than TS mRNA. BBR treatment reversed the resistance of the cells to cisplatin and BESpm (Marverti et al., 2013).

\subsection{BBR effects on signaling pathways implicated in drug resistance}

Combining BBR with signal transduction pathway inhibitors can inhibit the drug resistance of certain cancers. BBR can interact with the epidermal growth factor receptor (EGFR)/human epidermal growth factor receptor (HER2) inhibitor lapatinib and suppress the resistance of HER2 + breast cancer (Zhang et al., 2016). In lapatinib-resistant cells, lapatinib induces c-MYC/pro-NRF2 and GSK3beta signaling pathways. This results in stabilization of NRF2. In contrast, BBR treatment prevents some aspects of the growth promoting effects of lapatinib such as the induction of c-MYC expression. The combination of BBR and lapatinib decreased c-MYC expression, lapatinib-resistance and cell growth.

BBR inhibited the NF- $\mathrm{\kappa B}$ and the STAT3 pathways in cholangiocarcinoma. This induced cell cycle arrest at the $\mathrm{G}_{1}$ phase which suppressed cancer cell growth (Puthdee et al., 2017). Reduced CYCLIN D1 and CYCLIN E levels and increased levels of activated STAT3 and ERK1/2 were observed.

BBR also inhibits EGFR signaling and increased the effects of the EGFR inhibitors erlotinib and cetuximab in gastric cancer cells, both in in vitro and in vivo xenograft studies. These results are important as cetuximab plus chemotherapy has been examined in phase 2 clinical trials, however, the combination alone did not provide enough benefit for justification of performing phase 3 clinical trials. BBR was determined to inhibit activation of EGFR in tumors and the combination of BBR and cetuximab was more effective than treatment with either agent by itself. Part of the biochemical mechanism for growth inhibition resulted from decreased phosphorylation of STAT3 as well as decreased expression of BCLXL and cyclin D1 (Wang et al., 2016).

The PI3K/PTEN/AKT/mTORC1/GSK-3 signaling pathway is often regulated by nutraceuticals such as BBR. This pathway plays critical roles in: diabetes, cardiovascular diseases, inflammation, neuropathology, obesity, as well as cancer (McCubrey et al., 2017; Candido et al., 2018; Duda et al., 2018; McCubrey et al., 2018).

BBR affected AKT expression and its effects on EMT (Kou et al., 2016) by suppressing the migratory and invasive abilities of B16 melanoma cells. BBR altered the pluripotency of EMT-associated factors. BBR treatment suppressed phosphorylation of PI3K and AKT and downregulated retinoic acid receptor alpha (RARalpha) while increasing RARbeta and RARgamma expression. The PI3K inhibitor, LY294002, which will suppress AKT activity, had similar effects as BBR on most of the examined biochemical properties, except for RARgamma regulation. Importantly, BBR could reverse EMT in B16 melanoma cells.

BBRs also has effects on the RAF/MEK/ERK signaling pathway. BBR inhibited the proliferation of MGC 803 gastric cancer cells in vitro and in in vivo xenograft studies in mice (Li et al., 2016). BBR was determined to inhibit interleukin-8 (IL-8) expression. BBR affected $\mathrm{p} 38^{\mathrm{MAPK}}$, ERK and c-Jun N-terminal kinase (JNK) expression.

BBR altered the activity of the hedgehog $(\mathrm{Hh})$ signaling pathway in medulloblastoma cells. Some medulloblastoma cells may exhibit growth addiction to the Hh signaling as their proliferation is dependent on activation of this pathway. BBR inhibited the Hh pathway in these cells by suppressing SMO activity. BBR was determined to inhibit Hh-dependent growth both in vitro and in vivo (Wang et al., 2015).

\subsection{BBR effects on miRs}

BBR affected the cisplatin-sensitivity of A2780 ovarian cancer cells. The miR-93/PTEN/AKT signaling pathway was determined to be important in the induction of apoptosis and $\mathrm{G}_{0} / \mathrm{G}_{1}$ arrest. BBR treatment resulted in decreased levels of miR-93 cisplatin-resistant cells which reulted in their conversion to cisplatin-sensitive cells. In the absence of miR-93, PTEN tumor suppressor activity reappeared and inhibited AKT activity and apoptosis occurred. These studies established a regulatory link between miR-93, PTEN and AKT which could be modified by BBR treatment. BBR treatment may also eliminate the effects that miR-93 has on PTEN and relieve cisplatin-resistance in ovarian cancer cells (Chen et al., 2015).

BBR treatment an also relieve the cisplatin-resistance of gastric cancer cells by upregulation of miR-203 and suppression of the 
anti-apoptotic protein BCLW. miR-203 can bind mRNAs encoding BCLW and inhibit their expression and induce apoptosis (You et al., 2016). BBR can inhibit drug transporter expression in MCF-7 breast cancer cells. The expression of drug transporters is associated with both drug resistance and cancer stem cells (Kim et al., 2008).

BBR can also relieve the cisplatin-resistance of ovarian cancer cells by inhibiting the expression of miR-21. This occurred in part by loss of inhibition of the programmed cell death 4 (PDCD4) tumor suppressor gene (Liu et al., 2013). Previously it had been shown that in glioblastoma cells, miR-21 effected negatively the expression of matrix metalloproteinase (MMP) inhibitors, namely reversion inducing cysteine rich protein with kazal motifs (RECK) and tissue inhibitor of metalloproteinases (TIMP) (Gabriely et al., 2008). This can affect MMP9 expression and invasion.

BBR also suppressed miR-21 expression in HCT116 CRC cells (Lï et al., 2018). This resulted in decreased cancer cell viability and increased apoptosis. BBR also increased integrin subunit beta 4 (ITG $\beta 4$ ) and PDCD4 expression in these cells. BBR can also suppress miR-21 expression in glioma cells (Luo et al., 2017). miR-21 can promote invasion and migration in glioma cells by activation of SOX2 and $\beta$-catenin signaling.

Thus, metformin and BBR can relieve the drug resistance of cancer cells by various mechanisms. This is a rapidly emerging field and we have described pathways regulated by metformin and BBR. The regulation of miR expression by metformin and BBR may play critical roles in relieving chemotherapeutic drug resistance.

\subsection{Chemically-modified BBRs_Enhancing effects of BBR by medicinal chemistry}

We have recently reviewed various modified BBR compounds that have been developed (McCubrey et al., 2017; Abrams et al., 2018; Abrams et al., 2019). In this current manuscript, we will summarize some novel advances in the field. Various medicinal chemical approaches have been made to design more active BBRs and other nutraceuticals (Bhowmik et al., 2012b; Guamán Ortiz et al., 2014; Bhowmik and Kumar, 2016). NO-donating proto-BBRs have been developed which display more potent activity against certain tumor cells such as HepG2 hepatocellular carcinoma cells (Chen et al., 2017). These compounds release NO and the antitumor effects of certain compounds could be inhibited by a NO scavenger. Compound 15a developed by this group exhibited a broad anti-tumor efficacy and was selective against hepatocellular tumor cells as opposed to normal liver LO-2 cells. Compound 15a was determined to block HepG2 cells in the G2 phase of the cell cycle and induce apoptosis.

A panel of position-13 substituted BBRs was developed by Naxospharma in Milan Italy (Bhowmik et al., 2012b; Tillhon et al., 2012; Pierpaoli et al., 2013; Guaman Ortiz et al., 2014a; Guaman Ortiz et al., 2014b; Guaman Ortiz et al., 2015; Pierpaoli et al., 2015; Pierpaoli et al., 2018). These compounds exhibit enhanced activity in comparison to BBR against various cancer cells including colon
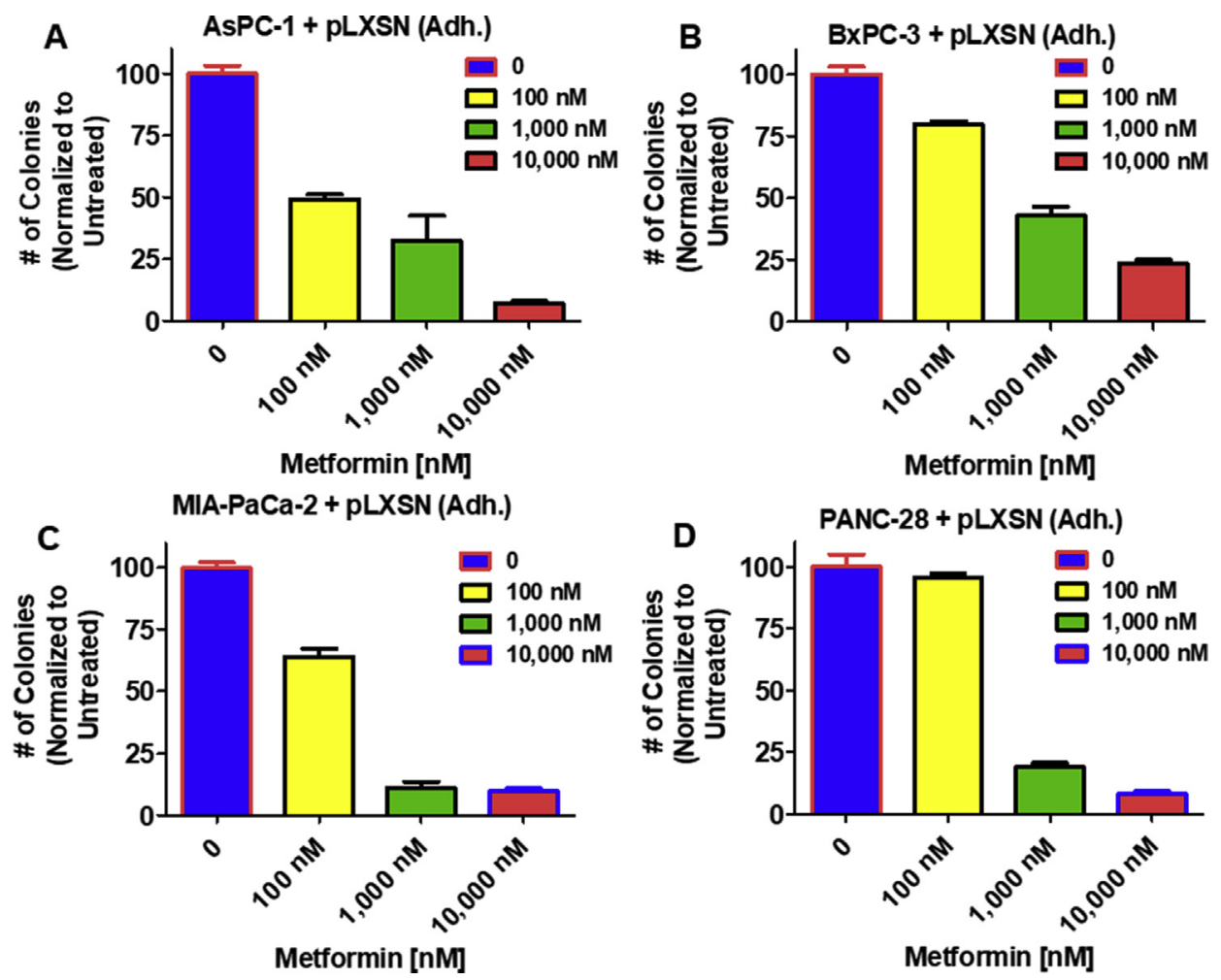

Fig. 3. Effects of Metformin on Colony Formation in Four PDAC Cell Lines. The effects of different concentrations of metformin on the colony forming ability of: AsPC-1 + pLXSN (Adh.) (Panel A), BxPC-3 + pLXSN (Adh.) (Panel B), MIA-PaCa-2 + pLXSN (Adh.) (Panel C) and PANC$28+$ pLXSN (Adh.) (Panel D) cells are presented. Colonies were normalized with each drug-treated cell line to the control no drug treated plates. 

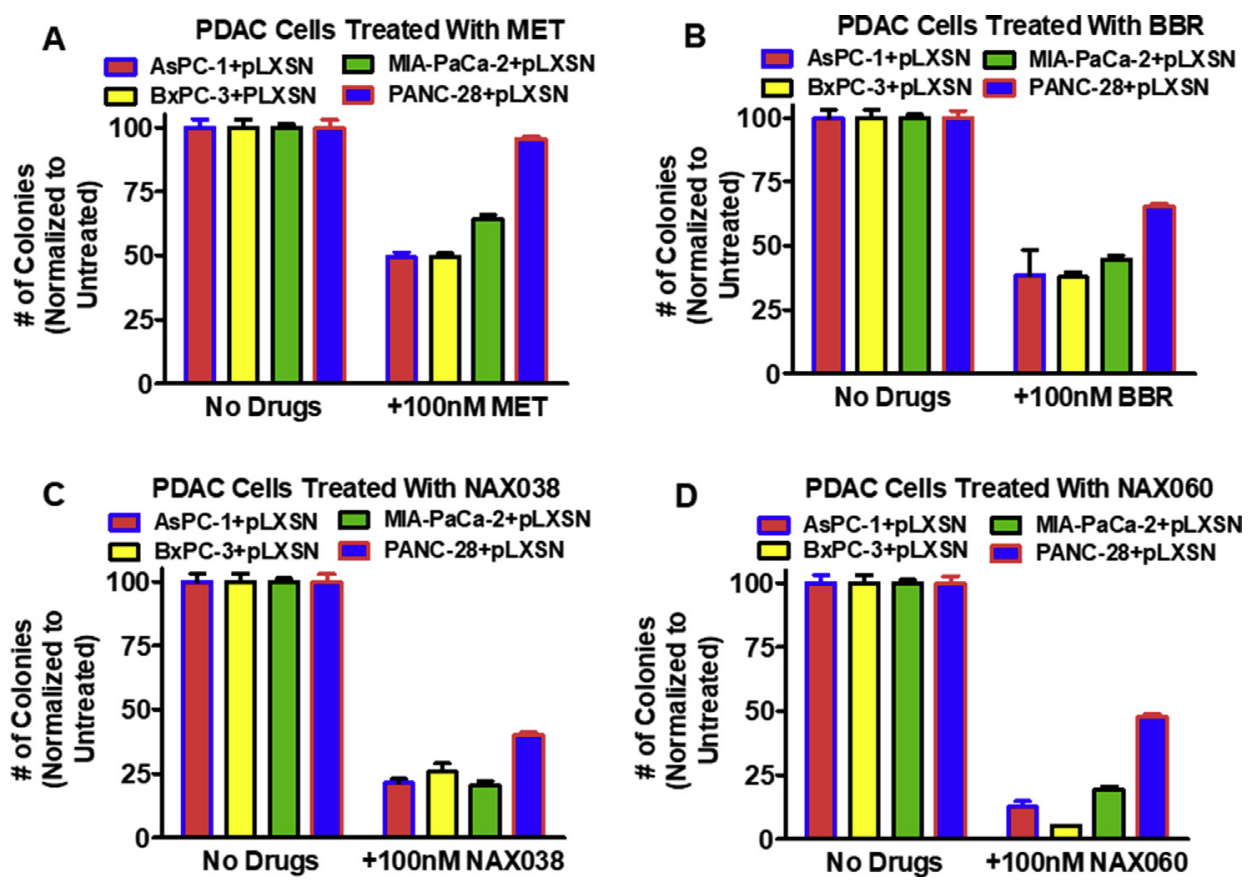

Fig. 4. Effects of $100 \mathrm{nM}$ Dose of Metformin, BBR, NAX038 and NAX060 on Colony Formation in Four PDAC Cell Lines. The effects of no drugs (all panels) or $100 \mathrm{nM}$ metformin (MET) (Panel A), $100 \mathrm{nM}$ BBR (Panel B), $100 \mathrm{nM}$ NAX038 (Panel C) and $100 \mathrm{nM}$ NAX060 (Panel D) on the colony forming ability of: AsPC-1 + pLXSN (Adh.) (red bars), BxPC-3 + pLXSN (Adh.) (yellow bars), MIA-PaCa-2 + pLXSN (Adh.) (green bars) and PANC-28 + pLXSN (Adh.) (blue bars) cells are presented. Colonies were normalized with each drug-treated cell line respectively, to the control no drug-treated plates.

and breast cancer (Guaman Ortiz et al., 2015; Pierpaoli et al., 2015; Pierpaoli et al., 2018).

BBR has a condensed polyaromatic buckled structure due to partial saturation in one of the rings and provides an attractive natural lead for the introduction of various chemical modifications. In this respect, the BBR derivatives (NAX compounds) developed by Naxospharma (Milan, Italy) bear (un)substituted(hetero)aromatic moieties bonded to position 13 of the parent alkaloid skeleton through a hydrocarbon linker of variable length, in a fashion to possibly create a geometric propensity for additional stacking-type, non-covalent aromatic interactions with cellular targets. Aromatic interactions are ubiquitous in nature and their geometry plays a central role in the molecular interaction with biological macromolecules. The 13-phenylalkyl and 13-diphenylalkyl BBR derivative NAX compounds were determined to result in telomeric DNA G-quadruplexes. The NAX042 and NAX053 compounds were examined in terms of telomeric G-quadruplexes in this system (Ferraroni et al., 2016). NAX042 and NAX053 were the best compounds in terms of simulating the interaction with human telomeric DNA G4 structures.

In the following studies, we have investigated the abilities of NAX compounds to interact with metformin and inhibit the growth of PDAC cell lines. We have investigated the effects of the introduction of one phenyl moiety (NAX012, NAX042), two phenyl moieties (benzhydryl) (NAX035, NAX053) and one heterocycle (pyridine) (NAX075, NAX077). In the monophenyl series, we investigated also the effects of single or multiple electron-releasing (NAX038, NAX054) or electron-withdrawing (NAX014, NAX060, NAX111) substituents. The effects of two different lengths of the hydrocarbon linker were also examined. Some of the NAX compounds could interact with metformin and inhibit proliferation better than others. The presence of WT-TP53 was determined to influence the electiveness of certain NAX compounds upon co-treatment with metformin.

\section{Materials and methods}

\subsection{Cell lines and tissue culture}

The MIA-PaCa-2 PDAC (ATCC ${ }^{\oplus}$ CRM-CRL-1420 ${ }^{\mathrm{Tm}}$ ) was recovered from a 65 -year old Caucasian male PDAC patient (Deer et al., 2010). These cells were obtained from the ATCC (Rockville, MD, USA). The MDA-PANC-28 cell line (CVCL_3917) was isolated from a female patient (Frazier et al., 1996) and is often abbreviated PANC-28. PANC-28 cells were generously provided by Dr. Shrikanth A. G. Reddy, (MD Anderson Cancer Center, Houston, TX USA), where the PANC-28 cell line was isolated originally. The AsPC-1 PDAC cell line (ATCC ${ }^{\oplus}$ CRL-1682 ${ }^{\mathrm{TM}}$ ), was recovered from a 62-year old female PDAC patient (Deer et al., 2010). BxPC-3 cells were derived from a 61-year old PDAC patient (ATCC ${ }^{\circ}$ CRL-1687 ${ }^{\mathrm{TM}}$ ). BxPC-3 cells have mutant TP53 but WT KRAS (Deer et al., 2010).

Cells were cultured in Dulbecco's modified Eagles medium, (DMEM), (Invitrogen, Carlsbad, CA), antibiotics containing L-glutamine medium supplemented with $5 \%$ fetal bovine serum (FBS) purchased from Atlanta Biologicals, Atlanta, GA, USA) as described in 

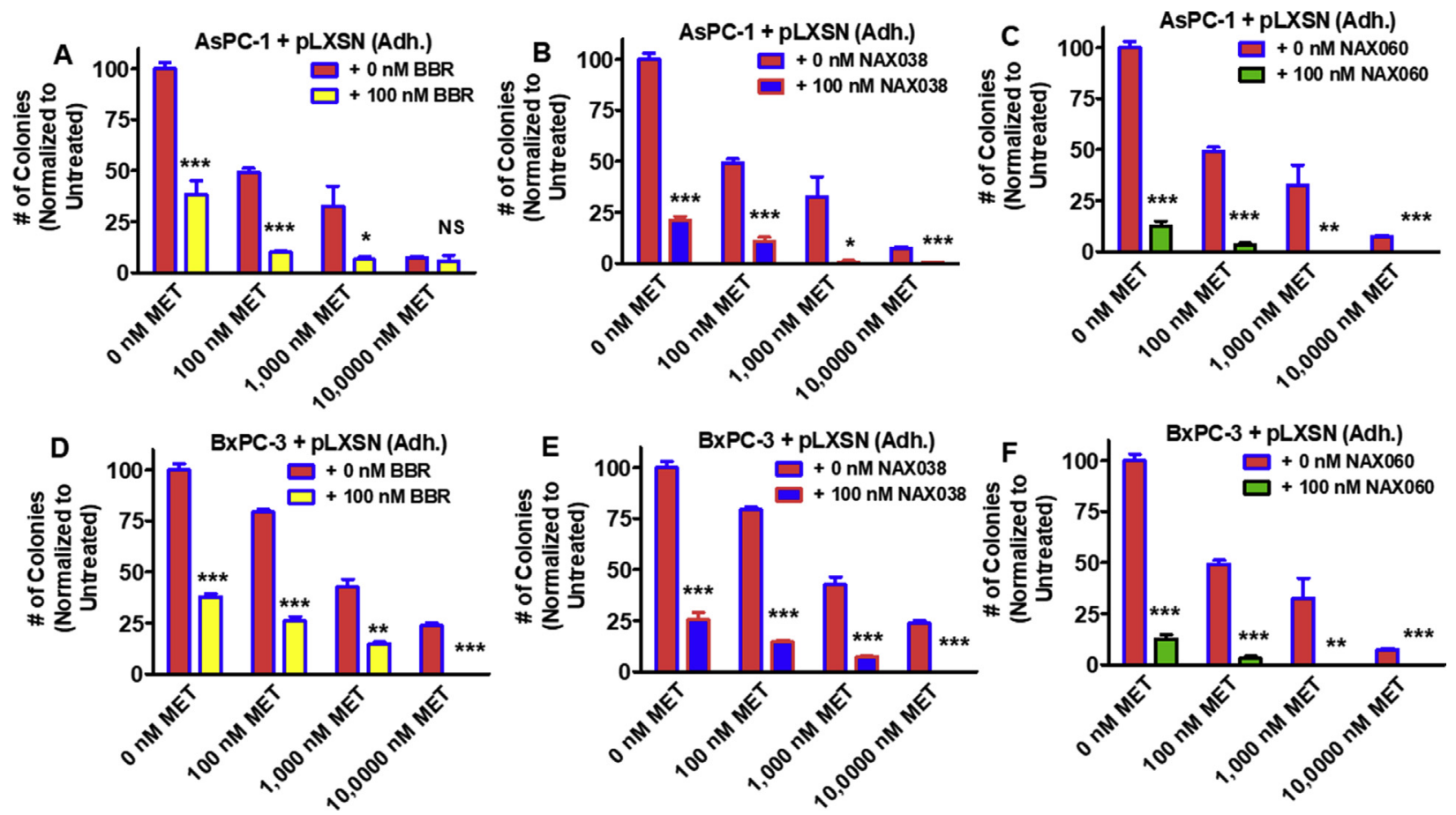

Fig. 5. Effects of Different Doses of Metformin in the Presence of No Drugs, $100 \mathrm{nM}$ Berberine, $100 \mathrm{nM}$ NAX038 or $100 \mathrm{nM}$ NAX060 on Colony Formation in AsPC-1 and BxPC-3 PDAC Cells. The effects of either no BBR or $100 \mathrm{nM}$ BBR on the colony formation in the presence of different doses of metformin (MET) in AsPC-1 + pLXSN (Adh.) (Panel A) and BxPC-3 + pLXSN (Adh) (Panel D) cells were determined. The effects of either no NAX038 or $100 \mathrm{nM}$ NAX038 on the colony formation in the presence of different doses of metformin (MET) in AsPC-1 + pLXSN (Adh.) (Panel B) and BxPC-3 + pLXSN (Adh) (Panel E) were determined. The effects of either no NAX060 or 100 nM NAX060 on the colony formation in the presence of different doses of metformin (MET) in AsPC-1 + pLXSN (Adh.) (Panel C) and BxPC-3 + pLXSN (Adh) (Panel F) cells were determined. Different treated cell lines were normalized to untreated respective cell line. Statistical analyses were performed in comparison with BBR, NAX038 or NAX060 treated cells vs. metformin-alone treated cells where appropriate by the Student T test on the means and standard deviations of various treatment groups. ${ }^{* * *}=P<0.0001,{ }^{*} * P<0.005,{ }^{*} P<0.05$, NS $=$ not statistically significant.

(Abrams et al., 2018a; Abrams et al., 2018b). NAX compounds were synthesized, purified and provided by our collaborator and coauthor, Dr. Paolo Lombardi (Naxospharma, Milan, Italy) (Guamán Ortiz et al., 2014, Guaman Ortiz et al., 2015; Pierpaoli et al., 2013, 2015, 2018). BBR was purchased from Sigma-Aldrich (Saint Louis, MO, USA).

\subsection{Infection of cells with a retroviral vector encoding WT-TP53}

PDAC cell lines were infected with either a retroviral vector encoding WT-TP53 or the empty pLXSN vector as a control as described (Abrams et al., 2018, 2019). Stably infected cell lines were isolated in the presence of $2 \mathrm{mg} / \mathrm{ml} \mathrm{G} 418$ (geneticin) SigmaAldrich. Pools were established after approximately four weeks in culture (Abrams et al., 2018, 2019).

\subsection{Methylthiazole tetrazolium assays}

Methylthiazole tetrazolium (MTT) assays were performed to determine the sensitivity of the pancreatic cancer cells to the modified NAX compounds and berberine as described (Abrams et al., 2018, 2019).

To test the hypothesis that the various $\mathrm{IC}_{50} \mathrm{~s}$ in the PDAC cells treated with the NAX compounds were statistically different than in the same cells treated the same day with the parental BBR, student's T tests were performed using Graph Pad Prism (QuickCals) statistical analysis.

\subsection{Colony formation assays}

Cells were seeded at 500 cells per well into three wells of six well Falcon plates (Falcon/Corning, Durham, NC, USA). Cells were treated with the indicated concentrations of chemotherapeutic drugs, metformin, BBR or NAX compounds. Colonies were propagated for approximately two weeks. When the colony size was approximately forty to 100 cells, the number of colonies were determined after giemsa staining of the colonies in the wells. The number of colonies were determined in three wells per condition and the average and standard deviation determined. The number of colonies in each condition were normalized to the untreated samples. 

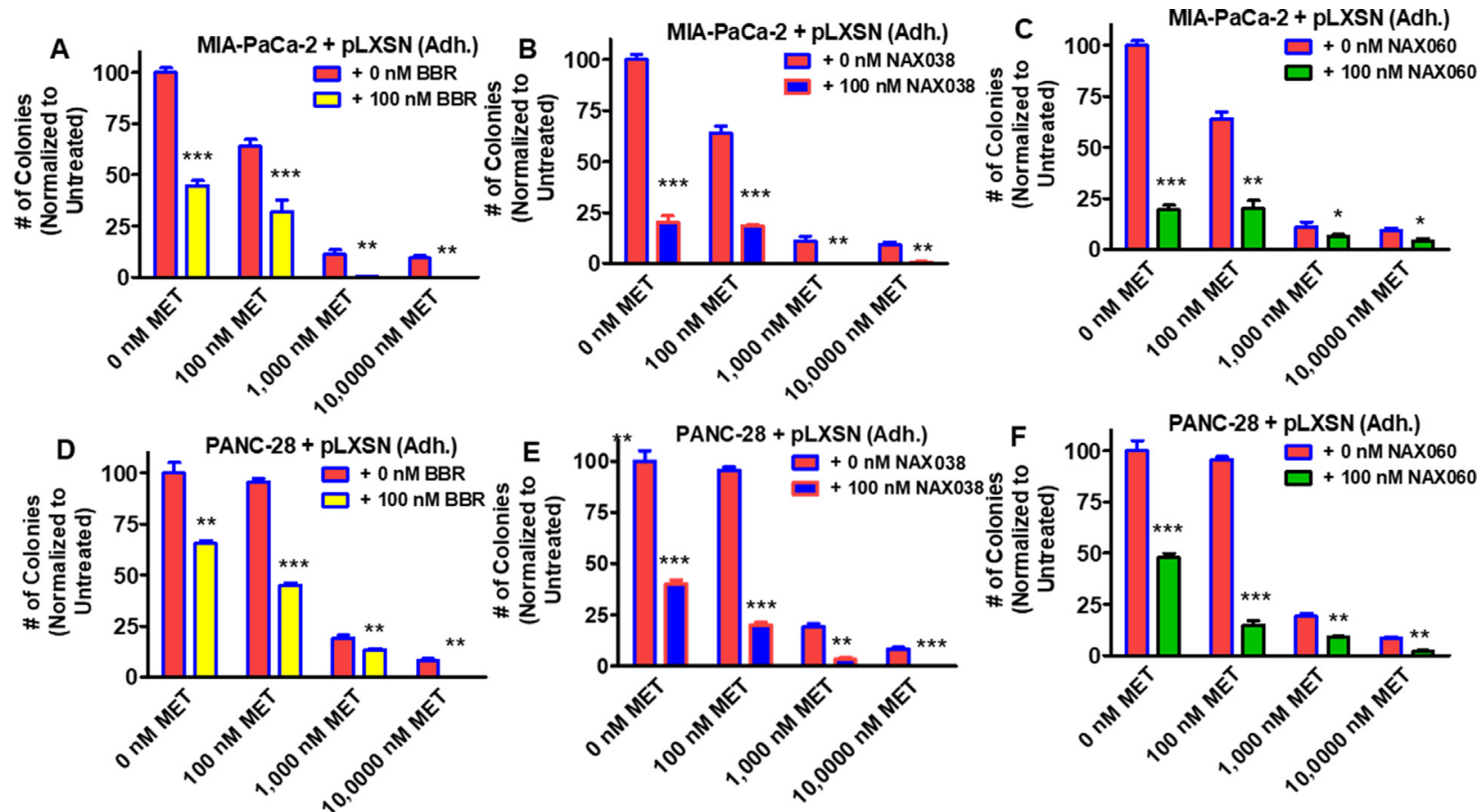

Fig. 6. Effects of Different Doses of Metformin in the Presence of No Drug, $100 \mathrm{nM}$ Berberine, $100 \mathrm{nM}$ NAX038 or $100 \mathrm{nM}$ NAX060 on Colony Formation in MIA-PaCa-2 and PANC-28 PDAC Cells. The effects of either no BBR or $100 \mathrm{nM}$ BBR on the colony formation in the presence of different doses of metformin (MET) in MIA-PaCa-2 + pLXSN (Adh.) (Panel A) and PANC-28 + pLXSN (Adh) (Panel D) were determined. The effects of either no NAX038 or $100 \mathrm{nM}$ NAX038 on the colony formation in the presence of different doses of metformin (MET) in MIA-PaCa$2+$ pLXSN (Adh.) (Panel B_ and PANC-28 + pLXSN (Adh.) (Panel E) were determined. The effects of either no NAX060 or 100 nM NAX060 on the colony formation in the presence of different doses of metformin (MET) in MIA-PaCa-2 + pLXSN (Adh.) (Panel C) and PANC-28 + pLXSN (Adh.) (Panel F) were determined. Different treated cell lines were normalized to untreated respective cell line. Statistical analyses were performed in comparison with BBR, NAX038 or NAX060 treated cells vs. metformin-alone treated cells where appropriate by the Student T test on the means and standard deviations of various treatment groups. ${ }^{* * *}=\mathrm{P}<0.0001,{ }^{* *} \mathrm{P}<0.005,{ }^{*} \mathrm{P}<0.05$, NS $=$ not statistically significant.

Statistics were performed as described above.

\section{Results}

\subsection{Effects of metformin on PDAC colony formation}

The effects of different doses of metformin on the colony formation of AsPC-1 + pLXSN (Panel A), BxPC-3 + pLXSN (Panel B), MIA-PaCa-2 + pLXSN (Panel C) and PANC-28 + pLXSN (Panel D) are presented in Fig. 3. These are four different PDAC cell lines. In these experiments, adherent (Adh.) cells were used. These cells had been infected with the pLXSN retrovirus as a control for retroviral infection as experiments presented in Figs. 9-15 and other studies were performed with cells infected with a WT-TP53 virus (Abrams et al., 2018, 2019). Dose-dependent effects of metformin were observed. In general, AsPC-1 and MIA-PaCa- 2 cells were more sensitive to metformin than either BxPC-3 or PANC-28 cells, however, the growth of all four PDAC lines was suppressed by over $50 \%$ when $1000 \mathrm{nM}$ and greater concentrations of metformin were used.

\subsection{Effects of low doses (100 nM) of metformin, BBR and NAX compounds on colony formation in PDAC cells}

For comparison purposes, a diagram of the effects of: $100 \mathrm{nM}$ metformin, $100 \mathrm{nM}$ BBR, $100 \mathrm{nM}$ NAX038 and $100 \mathrm{nM}$ NAX060 on the four different PDAC cell lines is present in Fig. 4. The PANC-28 cell line was the most resistant to $100 \mathrm{nM}$ metformin (Panel A). All four cell lines were more sensitive to either $100 \mathrm{nM}$ NAX038 or $100 \mathrm{nM}$ NAX060 (Panels C and D) than either $100 \mathrm{nM}$ metformin or $100 \mathrm{nM}$ BBR (Panels A and B).

\subsection{Effects of BBR, NAX038 and NAXO60 on different doxes of metformin on colony formation in four PDAC cell lines}

Figs. 5 and 6 document the effects of addition of different concentrations of metformin on the colony formation of AsPC1 + pLXSN (Adh.) (Fig. 5, Panels A-C), BxPC-3 + pLXSN (Adh.) (Fig. 5, Panels D-F), MIA-PaCa-2 + pLXSN (Adh.) (Fig. 6, Panel A-C) and PANC-28 + pLXSN (Adh.) (Fig. 6, Panels D-F). As presented previously, metformin inhibited colony formation in a dose- 

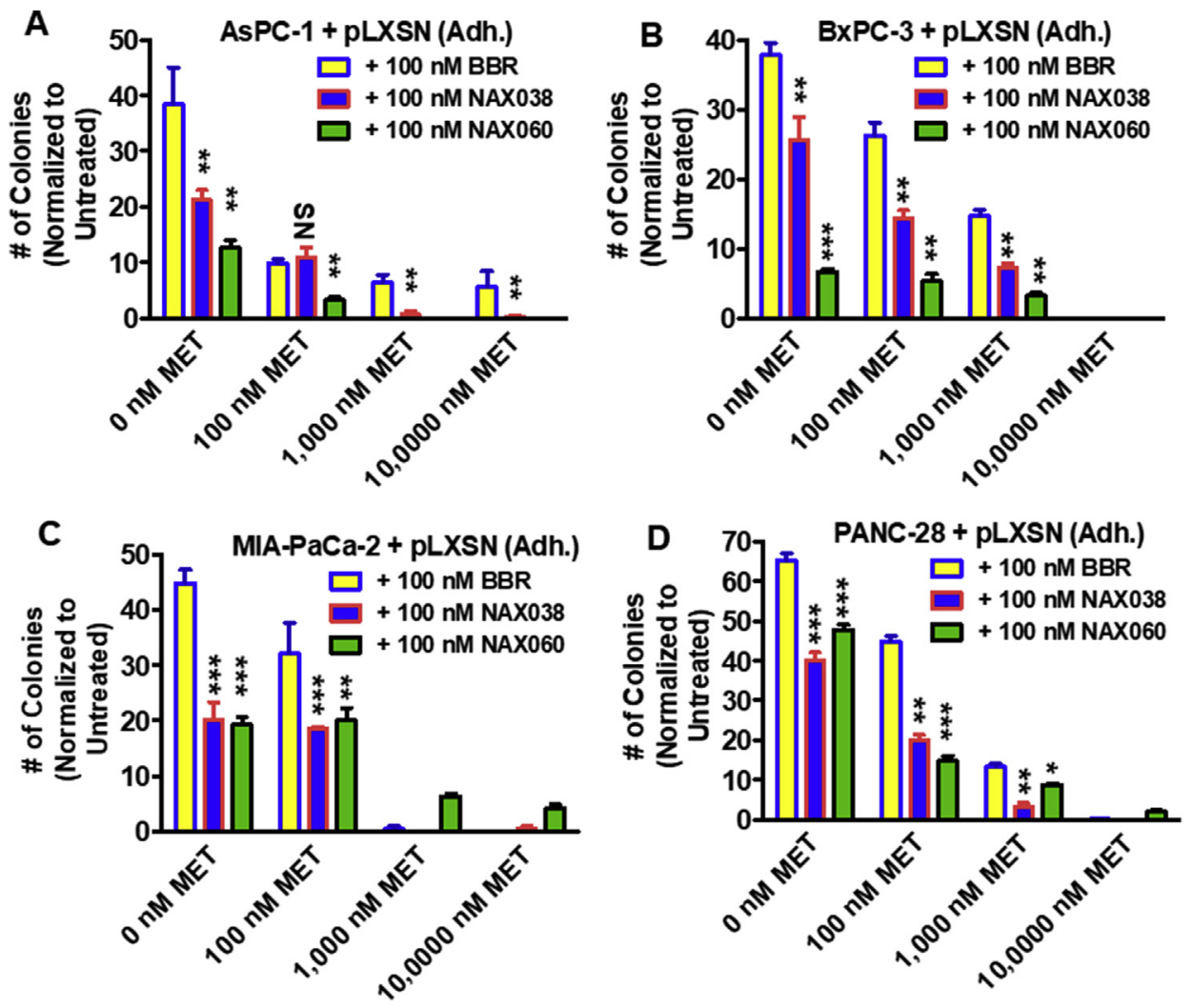

Fig. 7. Effects of 100 nM Dose of BBR, NAX038 and NAX060 on Colony Formation in the Presence of Different Concentrations of Metformin in Four PDAC Cell Lines. The effects of $100 \mathrm{nM}$ BBR (yellow bars) $100 \mathrm{nM}$ NAX038 (blue bars) and 100 nM NAX060 (green bars) on the colony forming ability of: AsPC-1 + pLXSN (Adh.) (Panel A), BxPC-3 + pLXSN (Adh.) (Panel B), MIA-PaCa-2 + pLXSN (Adh.) (Panel C) and PANC$28+$ pLXSN (Adh.) (Panel D) are presented. Colonies were normalized with each drug treated cell line to the control no drug treated plates. Statistical analyses were performed in relationship to metformin and NAX038 or NAX060 vs BBR treated where appropriate by the Student T test on the means and standard deviations of various treatment groups. ${ }^{* * *}=P<0.0001,{ }^{* *} P<0.005$, $* P<0.05$, NS $=$ not statistically significant.
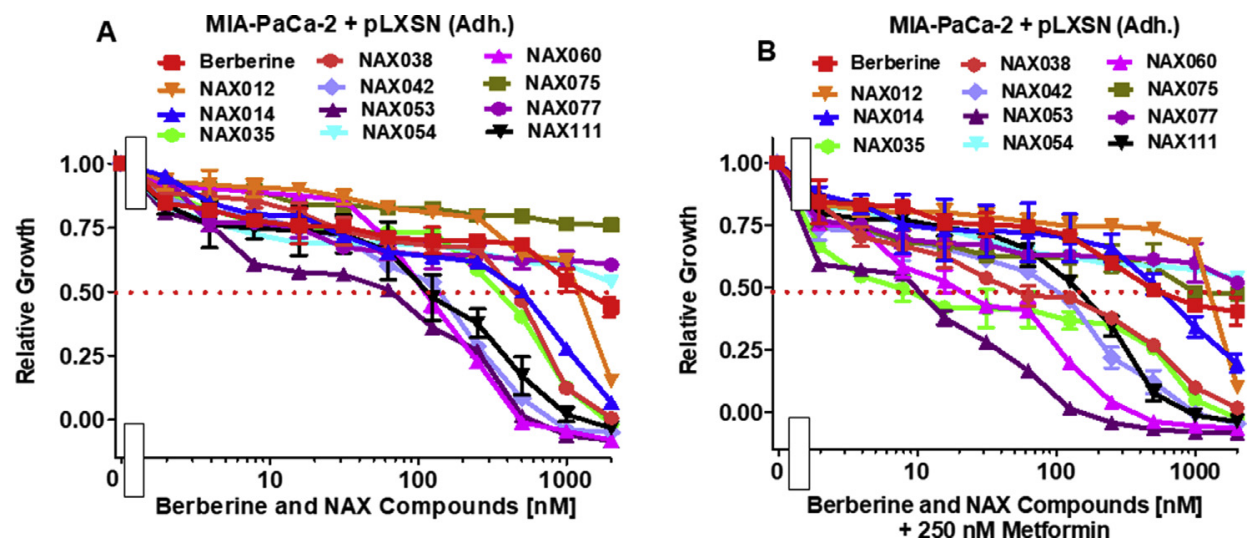

Fig. 8. Effects of the Nutraceuticals Berberine And NAX Compounds on the Proliferation and IC50s of MIA-PaCa-2 + pLXSN (Adh.) Cells Cultured in the Absence and Presence of $250 \mathrm{nM}$ Metformin. Panel A) MTT analysis of MIA-PaCa-2 + pLXSN (Adh.) cells cultured with BBR or the different NAX compounds. Panel B) MTT analysis of MIA-PaCa-2 + pLXSN (Adh.) cells exposed to the different NAX compounds in the presence of $250 \mathrm{nM}$ metformin. All the experiments indicated in this figure were performed on the same day. These experiments were repeated 3 times and similar results were obtained.

dependent fashion. Addition of $100 \mathrm{nM}$ BBR augmented the effects of metformin-mediated inhibition of colony formation in AsPC$1+$ pLXSN (Adh) (Fig. 5, Panel A), BxPC-3 + pLXSN (Adh.) (Fig. 5, Panel D), MIA-PaCa-2 + pLXSN (Adh.) (Fig. 6, Panel A) and PANC-28 + pLXSN (Adh.) (Fig. 6, Panel D).

The effects of $100 \mathrm{nM}$ NAX038 were also examined in combination with metformin. Addition of 100 nM NAX038 augmented the 

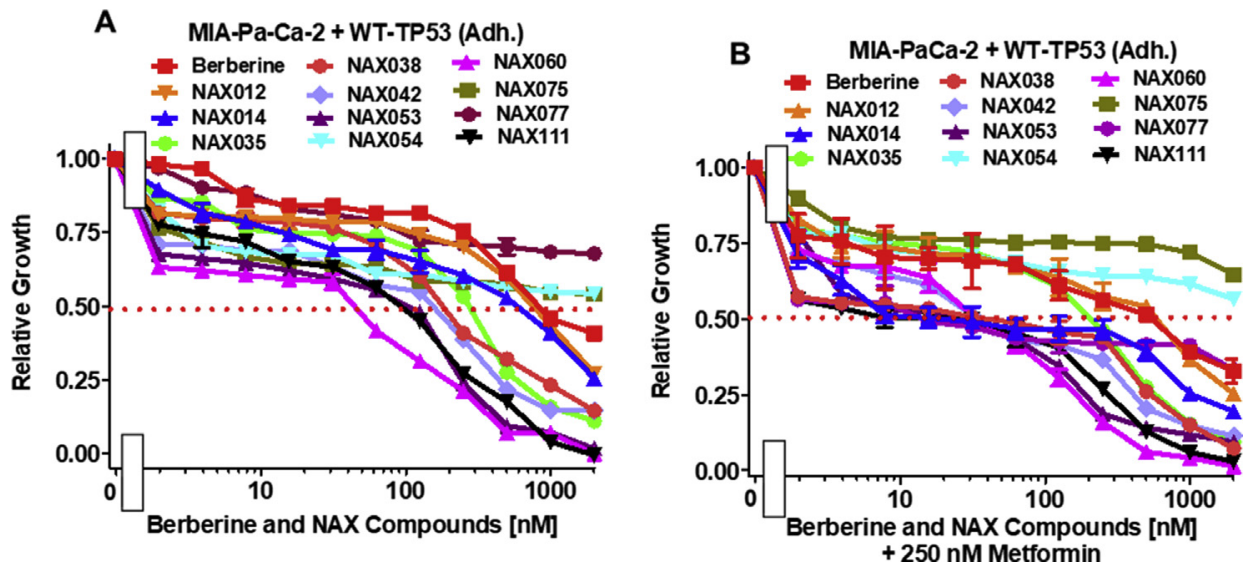

Fig. 9. Effects of the Nutraceuticals Berberine And NAX Compounds on the Proliferation and IC50s of MIA-PaCa-2 + WT-TP53 (Adh.) Cells Cultured in the Absence and Presence of 250 nM Metformin. Panel A) MTT analysis of MIA-PaCa-2 + WT-TP53 (Adh.) cells cultured with BBR or the different NAX compounds. Panel B) MTT analysis of MIA-PaCa-2 + WT-TP53 (Adh.) cells exposed to the different NAX compounds in the presence of $250 \mathrm{nM}$ metformin. All the experiments indicated in this figure were performed on the same day. These experiments were repeated 3 times and similar results were obtained.
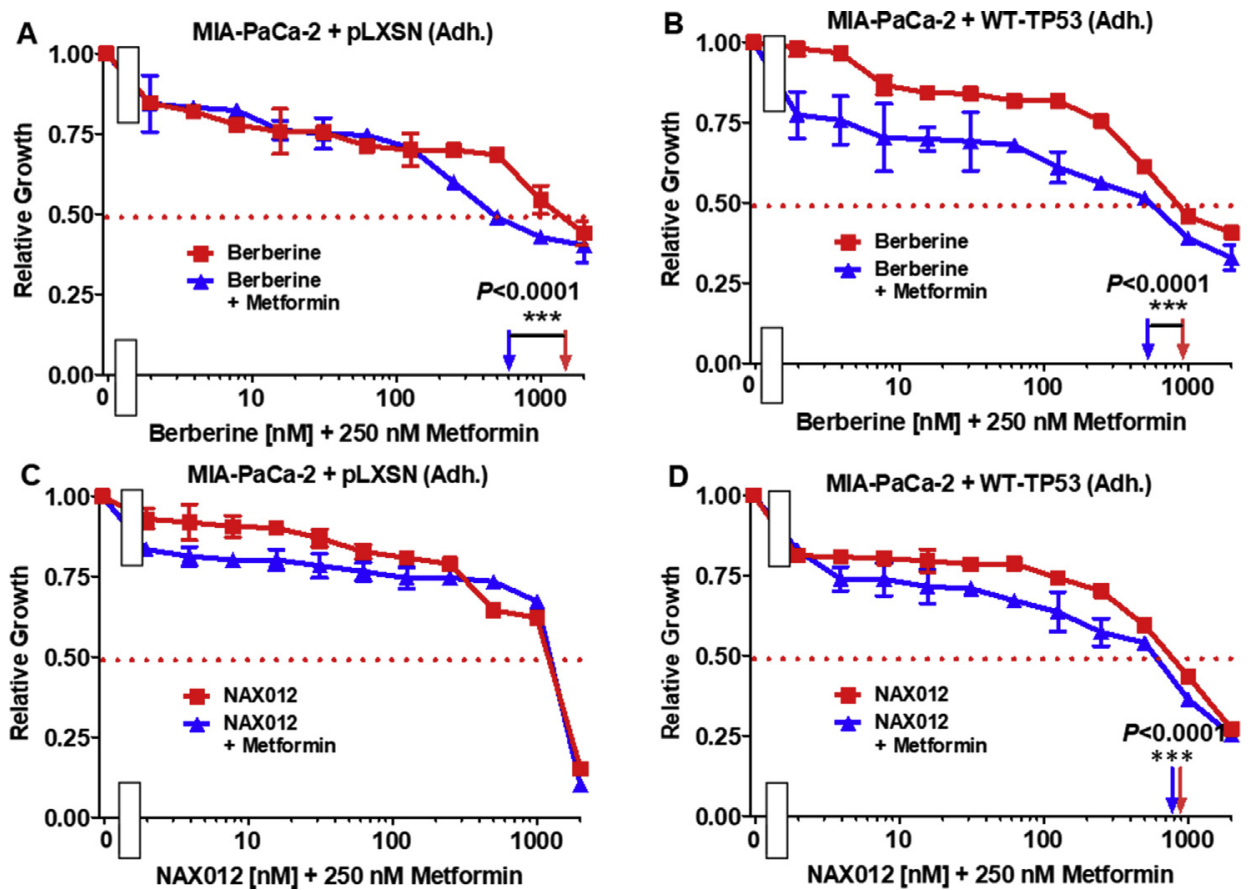

Fig. 10. Effects of the Nutraceutical Berberine and the NAX012 Compound on the Proliferation and IC50s of MIA-PaCa-2 + pLXSN (Adh.) and MIA-PaCa-2 + WT-TP53 (Adh.) Cells in the Absence and Presence of $250 \mathrm{nM}$ Metformin. Panel A) MTT analysis of MIA-PaCa-2 + pLXSN (Adh.) cells plated with different concentrations of berberine (solid red squares) or different concentrations of berberine and $250 \mathrm{nM}$ metformin (solid blue triangles). Panel B) MTT analysis of MIA-PaCa-2 + WT-TP53 (Adh.) cells plated with different concentrations of berberine (solid red squares) or different concentrations of berberine and $250 \mathrm{nM}$ metformin (solid blue triangles). Panel C) MTT analysis of MIA-PaCa-2 + pLXSN (Adh.) cells plated with different concentrations of NAX012 (solid red squares) or different concentrations of NAX012 and $250 \mathrm{nM}$ metformin (solid blue triangles). Panel D) MTT analysis of MIA-PaCa-2 + WT-TP53 (Adh.) cells plated with different concentrations of NAX012 (solid red squares) or different concentrations of NAX012 and $250 \mathrm{nM}$ metformin (solid blue triangles). All the experiments indicated in this figure were performed on the same day. These experiments were repeated 3 times and similar results were obtained.

effects of metformin-mediated inhibition of colony formation in AsPC-1 + pLXSN (Adh) (Fig. 5, Panel B), BxPC-3 + pLXSN (Adh.) (Fig. 5, Panel E), MIA-PaCa-2 + pLXSN (Adh.) (Fig. 6, Panel B) and PANC-28 + pLXSN (Adh.) (Fig. 6, Panel E).

The effects of $100 \mathrm{nM}$ NAX060 were also examined in combination with metformin. Addition of $100 \mathrm{nM}$ NAX060 augmented the effects of metformin-mediated inhibition of colony formation in AsPC-1 + pLXSN (Adh) (Fig. 5, Panel C), BxPC-3 + pLXSN (Adh.) (Fig. 5, Panel F), MIA-PaCa-2 + pLXSN (Adh.) (Fig. 6, Panel C) and PANC-28 + pLXSN (Adh.) (Fig. 6, Panel F). Addition of 100 nM 

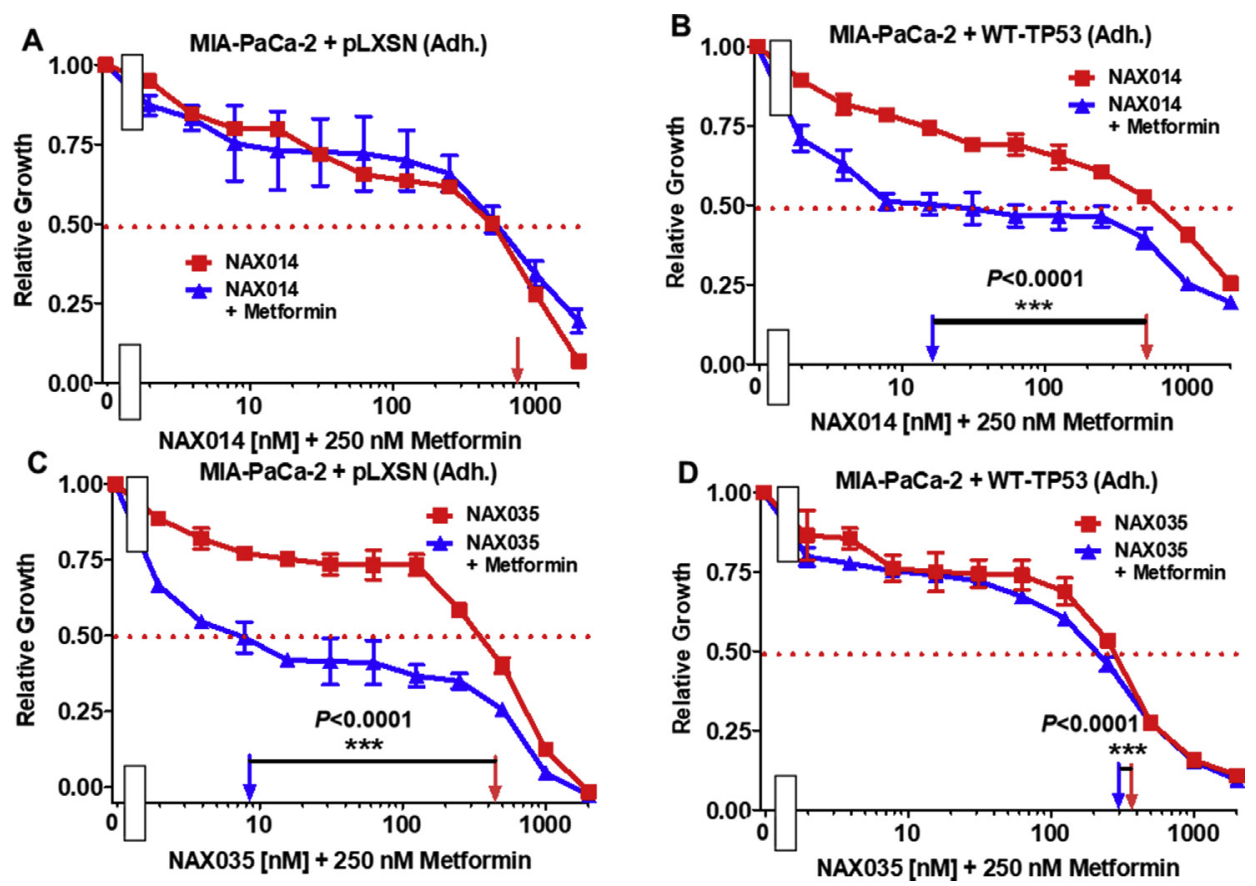

Fig. 11. Effects of the NAX014 and NAX035 Compounds on the Proliferation and IC50s of MIA-PaCa-2 + pLXSN (Adh.) and MIA-PaCa-2 + WT-TP53 (Adh.) Cells in the Absence and Presence of $250 \mathrm{nM}$ Metformin. Panel A) MTT analysis of MIA-PaCa-2 + pLXSN (Adh.) cells plated with different concentrations of NAX014 (solid red squares) or different concentrations of NAX014 and $250 \mathrm{nM}$ metformin (solid blue triangles). Panel B) MTT analysis of MIA-PaCa-2 + WT-TP53 (Adh.) cells plated with different concentrations of NAX014 (solid red squares) or different concentrations of NAX014 and $250 \mathrm{nM}$ metformin (solid blue triangles). Panel C) MTT analysis of MIA-PaCa-2 + pLXSN (Adh.) cells plated with different concentrations of NAX035 (solid red squares) or different concentrations of NAX012 and 250 nM metformin (solid blue triangles). Panel D) MTT analysis of MIA-PaCa-2 + WT-TP53 (Adh.) cells plated with different concentrations of NAX035 (solid red squares) or different concentrations of NAX035 and $250 \mathrm{nM}$ metformin (solid blue triangles). All the experiments indicated in this figure were performed on the same day. These experiments were repeated 3 times and similar results were obtained.

BBR, NAX038 and NAX060 augmented the ability of metformin to inhibit colony formation in AsPC-1 + pLXSN (Adh.) (Fig. 5, Panel C), BXPC-3 + pLXSN (Adh.) (Fig. 5, Panel F), MIA-PaCa-2 + pLXSN (Adh.) (Fig. 6, Panel C) and PANC-28 + pLXSN (Adh.) cells (Panel 6, Panel F).

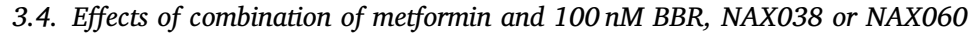

A more condensed depiction of the effects of BBR, NAX038 and NAX060 on different concentrations of metformin is presented in

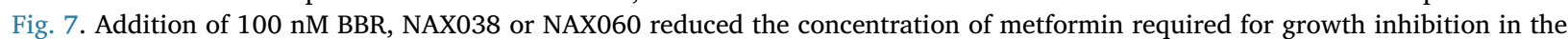
four different cell lines. In general, NAX038 and NAX060 suppressed proliferation in AsPC-1 + pLXSN (Adh.) and BxPC-3 + pLXSN (Adh.) in combination with metformin more than BBR (Fig. 7, Panels A and B). 100 nM NAX038 and NAX060 suppressed colony formation more than BBR in MIA-PaCa-2 + pLXSN (Adh.) when the cells were treated 0 and $100 \mathrm{nM}$ metformin (Fig. 7, Panel C). 100 nM NAX038 and NAX060 suppressed colony formation more than BBR when the PANC-28 + pLXSN (Adh.) were treated with 0 , 100 and $1000 \mathrm{nM}$ metformin (Fig. 7, Panel D).

3.5. Titrations of MIA-PaCa-2 + pLXSN and MIA-PaCa-2 + WT-TP53 cells with berberine, NAX compounds and a Sub-IC 50 dose of 250 nM metformin

The effects of titering MIA-PaCa-2 + pLXSN and MIA-PaCa-2 + WT-TP53 cells with different concentration of BBR, NAX compounds either by themselves (Figs. 8 and 9, A Panels) or also with $250 \mathrm{nM}$ metformin (Figs. 8 and 9, B Panels) were determined by MTT analysis. In addition, the effects of the BBR and NAX compounds were plotted separately (Figs. 10-15). The differences in the effects of the various NAX compounds in the presence and absence of $250 \mathrm{nM}$ metformin are summarized in Table 1 and Table 2.

As observed in Fig. 10, Panel A, the IC $_{50}$ for BBR in MIA-PaCa-2 + pLXSN (Adh) cells was approximately 1600 nM. Addition of $250 \mathrm{nM}$ metformin resulted in a 2.7 -fold decrease in the $\mathrm{IC}_{50}$ to $600 \mathrm{nM}$. The $\mathrm{IC}_{50}$ for BBR in MIA-PaCa-2 + WT-TP53 (Adh) cells was approximately $900 \mathrm{nM}$. Addition of $250 \mathrm{nM}$ metformin resulted in a 1.8-fold decrease in the IC 50 to $500 \mathrm{nM}$ (Fig. 10 , Panel B).

The IC $_{50}$ for NAX012 in MIA-PaCa-2 + pLXSN (Adh) cells was approximately $1300 \mathrm{nM}$ Addition of $250 \mathrm{nM}$ metformin did not result in a change in the $\mathrm{IC}_{50}$ (Fig. 10, Panel C). The IC $_{50}$ for NAX012 in MIA-PaCa-2 + WT-TP53 (Adh) cells was approximately $900 \mathrm{nM}$. Addition of $250 \mathrm{nM}$ metformin resulted in a 1.6 -fold decrease in the $\mathrm{IC}_{50}$ to $550 \mathrm{nM}$ (Fig. 10, Panel D). 
A
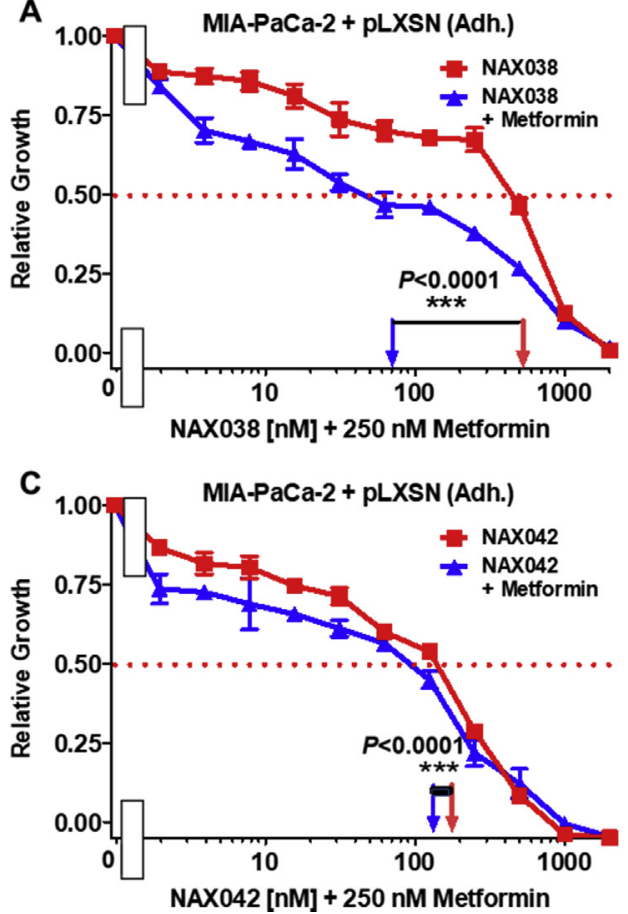
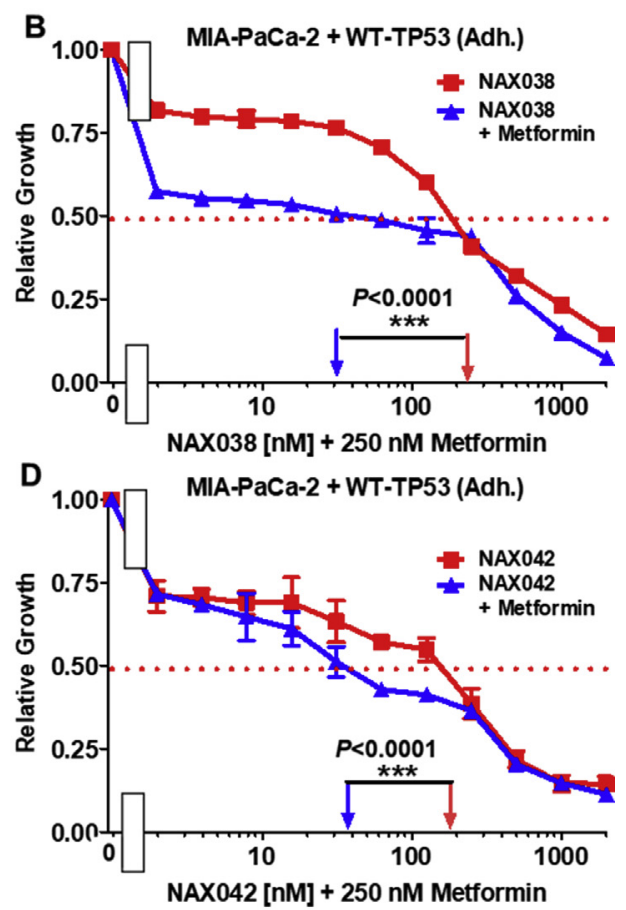

Fig. 12. Effects of the NAX038 and NAX042 Compounds on the Proliferation and IC50s of MIA-PaCa-2 + pLXSN (Adh.) and MIA-PaCa-2 + WT-TP53 (Adh.) Cells in the Absence and Presence of $250 \mathrm{nM}$ Metformin. Panel A) MTT analysis of MIA-PaCa-2 + pLXSN (Adh.) cells plated with different concentrations of NAX038 (solid red squares) or different concentrations of NAX038 and $250 \mathrm{nM}$ metformin (solid blue triangles). Panel B) MTT analysis of MIA-PaCa-2 + WT-TP53 (Adh.) cells plated with different concentrations of NAX038 (solid red squares) or different concentrations of NAX038 and $250 \mathrm{nM}$ metformin (solid blue triangles). Panel C) MTT analysis of MIA-PaCa-2 + pLXSN (Adh.) cells plated with different concentrations of NAX042 (solid red squares) or different concentrations of NAX042 and $250 \mathrm{nM}$ metformin (solid blue triangles). Panel D) MTT analysis of MIA-PaCa-2 + WT-TP53 (Adh.) cells plated with different concentrations of NAX042 (solid red squares) or different concentrations of NAX042 and $250 \mathrm{nM}$ metformin (solid blue triangles). All the experiments indicated in this figure were performed on the same day. These experiments were repeated 3 times and similar results were obtained.

The IC $_{50}$ for NAX014 in MIA-PaCa-2 + pLXSN (Adh) cells was approximately $750 \mathrm{nM}$ Addition of $250 \mathrm{nM}$ metformin did not result in a change in the $\mathrm{IC}_{50}$ (Fig. 11, Panel A). The $\mathrm{IC}_{50}$ for NAX014 in MIA-PaCa-2 + WT-TP53 (Adh) cells was approximately $500 \mathrm{nM}$. Addition of $250 \mathrm{nM}$ metformin resulted in a 33.3-fold decrease in the $\mathrm{IC}_{50}$ to $15 \mathrm{nM}$ (Fig. 11, Panel B).

The $\mathrm{IC}_{50}$ for NAX035 in MIA-PaCa-2 + pLXSN (Adh) cells was approximately $450 \mathrm{nM}$ Addition of $250 \mathrm{nM}$ metformin resulted in a 52.9-fold decrease in the IC $_{50}$ to $8.5 \mathrm{nM}$ (Fig. 11, Panel C). The IC 50 for NAX035 in MIA-PaCa-2 + WT-TP53 (Adh) cells was approximately $400 \mathrm{nM}$. Addition of $250 \mathrm{nM}$ metformin resulted in a 1.3-fold decrease in the $\mathrm{IC}_{50}$ to $300 \mathrm{nM}$ (Fig. 11, Panel D).

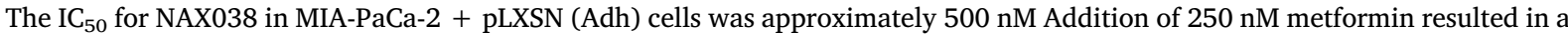
6.4-fold decrease in the IC $_{50}$ to $70 \mathrm{nM}$ (Fig. 12, Panel A). The IC $_{50}$ for NAX038 in MIA-PaCa-2 + WT-TP53 (Adh) cells was approximately $220 \mathrm{nM}$. Addition of $250 \mathrm{nM}$ metformin resulted in a 7.3-fold decrease in the $\mathrm{IC}_{50}$ to $300 \mathrm{nM}$ (Fig. 12, Panel B).

The IC $_{50}$ for NAX042 in MIA-PaCa-2 + pLXSN (Adh) cells was approximately $180 \mathrm{nM}$ Addition of $250 \mathrm{nM}$ metformin resulted in a 1.2-fold decrease in the $\mathrm{IC}_{50}$ to $150 \mathrm{nM}$ (Fig. 12, Panel C). The $\mathrm{IC}_{50}$ for NAX042 in MIA-PaCa-2 + WT-TP53 (Adh) cells was approximately $200 \mathrm{nM}$. Addition of $250 \mathrm{nM}$ metformin resulted in a 5-fold decrease in the $\mathrm{IC}_{50}$ to $40 \mathrm{nM}$ (Fig. 12, Panel D).

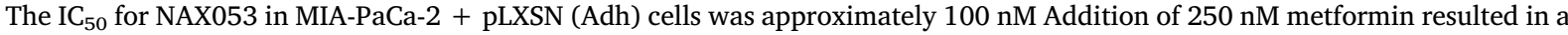
6.7-fold decrease in the $\mathrm{IC}_{50}$ to $15 \mathrm{nM}$ (Fig. 13, Panel A). The IC 50 for NAX053 in MIA-PaCa-2 + WT-TP53 (Adh) cells was approximately $130 \mathrm{nM}$. Addition of $250 \mathrm{nM}$ metformin resulted in a 3.3-fold decrease in the IC 50 to $40 \mathrm{nM}$ (Fig. 13, Panel B).

The IC $_{50}$ for NAX054 in MIA-PaCa-2 + pLXSN (Adh) cells was approximately $2000 \mathrm{nM}$ Addition of $250 \mathrm{nM}$ metformin did not result in a decrease in the $\mathrm{IC}_{50}$ (Fig. 13, Panel C). The $\mathrm{IC}_{50}$ for NAX054 in MIA-PaCa-2 + WT-TP53 (Adh) cells was $>2000 \mathrm{nM}$. Addition of $250 \mathrm{nM}$ metformin resulted in a slight decrease in the $\mathrm{IC}_{50}$ to $2000 \mathrm{nM}$ (Fig. 13, Panel D).

The $\mathrm{IC}_{50}$ for NAX060 in MIA-PaCa-2 + pLXSN (Adh) cells was approximately $180 \mathrm{nM}$ Addition of $250 \mathrm{nM}$ metformin resulted in a 6.4-fold decrease in the $\mathrm{IC}_{50}$ to $28 \mathrm{nM}$ (Fig. 14, Panel A). The IC 50 for NAX060 in MIA-PaCa-2 + WT-TP53 (Adh) cells was approximately $60 \mathrm{nM}$. Addition of $250 \mathrm{nM}$ metformin resulted in a 1.5-fold decrease in the $\mathrm{IC}_{50}$ to $40 \mathrm{nM}$ (Fig. 14, Panel B).

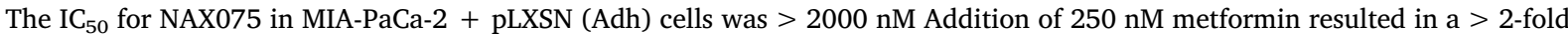
decrease in the $\mathrm{IC}_{50}$ to $1000 \mathrm{nM}$ (Fig. 14, Panel C). The IC $_{50}$ for NAX075 in MIA-PaCa-2 + WT-TP53 (Adh) cells was not observed in the absence and presence of $250 \mathrm{nM}$ (Fig. 14, Panel D).

The IC $_{50}$ for NAX077 in MIA-PaCa-2 + pLXSN (Adh) cells was $>2000 \mathrm{nM}$ Addition of $250 \mathrm{nM}$ metformin resulted in a $>2$-fold 

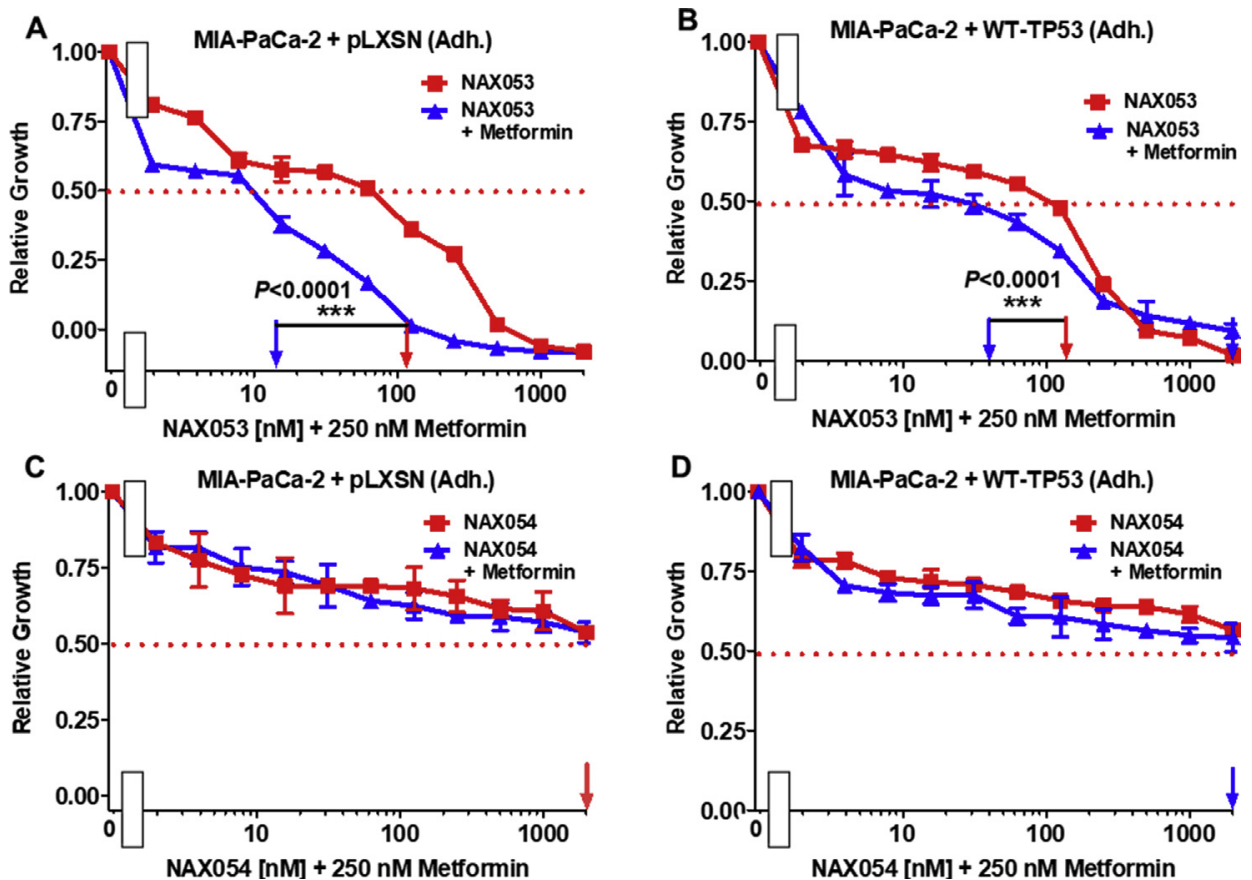

Fig. 13. Effects of the NAX053 and NAX054 Compounds on the Proliferation and IC50s of MIA-PaCa-2 + pLXSN (Adh.) and MIA-PaCa-2 + WT-TP53 (Adh.) Cells in the Absence and Presence of $250 \mathrm{nM}$ Metformin. Panel A) MTT analysis of MIA-PaCa-2 + pLXSN (Adh.) cells plated with different concentrations of NAX053 (solid red squares) or different concentrations of NAX053 and $250 \mathrm{nM}$ metformin (solid blue triangles). Panel B) MTT analysis of MIA-PaCa-2 + WT-TP53 (Adh.) cells plated with different concentrations of NAX054 (solid red squares) or different concentrations of NAX053 and $250 \mathrm{nM}$ metformin (solid blue triangles). Panel C) MTT analysis of MIA-PaCa-2 + pLXSN (Adh.) cells plated with different concentrations of NAX053 (solid red squares) or different concentrations of NAX054 and $250 \mathrm{nM}$ metformin (solid blue triangles). Panel D) MTT analysis of MIA-PaCa-2 + WT-TP53 (Adh.) cells plated with different concentrations of NAX054 (solid red squares) or different concentrations of NAX054 and $250 \mathrm{nM}$ metformin (solid blue triangles). All the experiments indicated in this figure were performed on the same day. These experiments were repeated 3 times and similar results were obtained.

decrease in the $\mathrm{IC}_{50}$ to $1000 \mathrm{nM}$ (Fig. 15, Panel A). The $\mathrm{IC}_{50}$ for NAX077 in MIA-PaCa-2 + WT-TP53 (Adh) cells was not observed upon addition with up to 2000. However, upon addition of $250 \mathrm{nM}$ metformin, an IC50 of approximately $20 \mathrm{nM}$ was observed, a decrease of $>100$-fold. (Fig. 15, Panel B).

The IC $_{50}$ for NAX111 in MIA-PaCa-2 + pLXSN (Adh) cells was approximately $200 \mathrm{nM}$ Addition of $250 \mathrm{nM}$ metformin did not result in a change in the IC $_{50}$ (Fig. 15, Panel C). The IC $_{50}$ for NAX11 in MIA-PaCa-2 + WT-TP53 (Adh) cells was approximately $150 \mathrm{nM}$. Addition of $250 \mathrm{nM}$ metformin resulted in a 16.8 -fold decrease in the $\mathrm{IC}_{50}$ to $9 \mathrm{nM}$ (Fig. 15, Panel D).

\section{Discussion}

Metformin is a commonly prescribed drug for the treatment of type-II diabetes. It is a glucose lowering drug which is given to diabetic patients in the absence of insulin treatment. Metformin effects the AMPK pathway which regulates TSC1/TSC2/RHEB/ mTORC1 signaling and metabolism, energy, redox and biomass (Candido et al., 2018). When AMPK is activated the pro-proliferative RAS/MEK/ERK and PI3K/PTEN/AKT/mTORC1 pathways are suppressed.

miRs have important effects on mitochondria (Li et al., 2012). One of the key targets of metformin is the mitochondria where it can alter bioenergetics (Andrzejewski et al., 2014). Metformin can have divergent effects on miR expression. Some studies have indicated that metformin may induce the expression of certain mIRs. Other investigations have indicated that metformin may suppress the expression of the same miR under different experimental conditions and divergent tissue types.

Metformin can have profound effects on mitochondrial dysfunction in breast cancer cells (Sharma \& Kumar., 2018). In these studies, metformin influenced the migration and induction of apoptosis in breast cancer cell lines. Metformin also altered the expression of many genes including: BCL2, BAX, MMP2, MMP9, miR-21 and miR-155.

Metformin can also have effects on drug-resistant prostate cancer cells by targeting mitochondrial complex-1. This was demonstrated to impair the proliferation and invasiveness of the drug-resistant PC3-DR cells but not drug-sensitive PC3 cells. Decreased expression of miR-205 is associated with drug resistance and EMT in PC3-DR cells. Induced re-expression of miR-205 was demonstrated to shift mitochondrial oxidative phosphorylation to Warburg metabolism and resulted in increased sensitivity to docetaxel (Ippolito et al., 2016).

Metformin can induce mitochondrial-regulated apoptosis in oral cancer cells. Metformin treatment of the oral cancer cell line KB1 


\section{A}
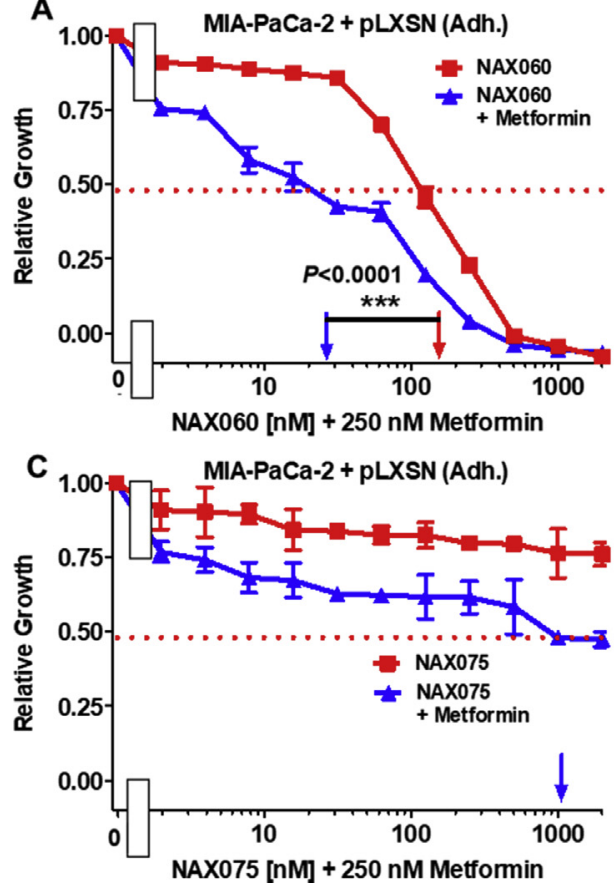
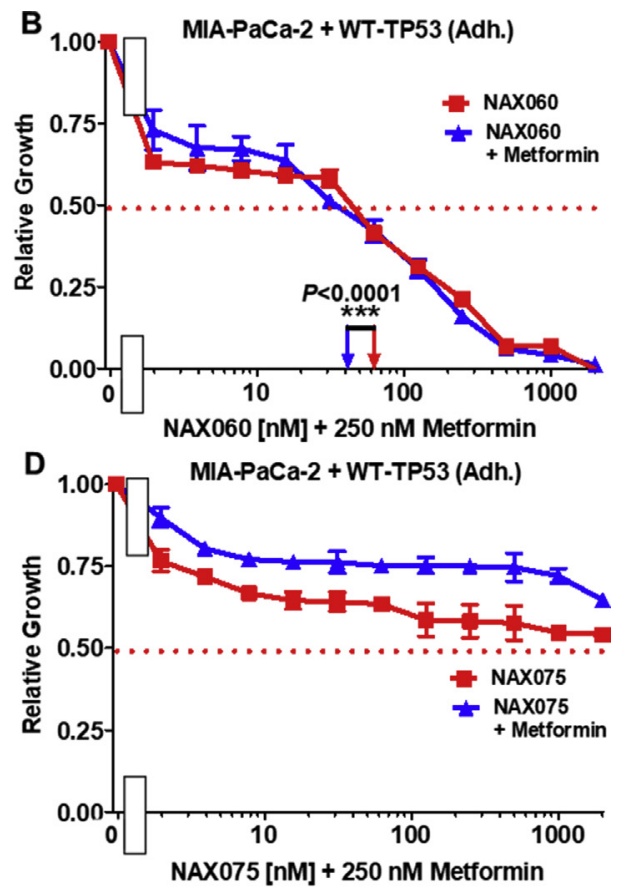

Fig. 14. Effects of the NAX060 and NAX075 Compounds on the Proliferation and IC50s of MIA-PaCa-2 + pLXSN (Adh.) and MIA-PaCa-2 + WT-TP53 (Adh.) Cells in the Absence and Presence of $250 \mathrm{nM}$ Metformin. Panel A) MTT analysis of MIA-PaCa-2 + pLXSN (Adh.) cells plated with different concentrations of NAX060 (solid red squares) or different concentrations of NAX060 and $250 \mathrm{nM}$ metformin (solid blue triangles). Panel B) MTT analysis of MIA-PaCa-2 + WT-TP53 (Adh.) cells plated with different concentrations of NAX060 (solid red squares) or different concentrations of NAX060 and $250 \mathrm{nM}$ metformin (solid blue triangles). Panel C) MTT analysis of MIA-PaCa-2 + pLXSN (Adh.) cells plated with different concentrations of NAX075 (solid red squares) or different concentrations of NAX075 and $250 \mathrm{nM}$ metformin (solid blue triangles). Panel D) MTT analysis of MIA-PaCa-2 + WT-TP53 (Adh.) cells plated with different concentrations of NAX075 (solid red squares) or different concentrations of NAX075 and $250 \mathrm{nM}$ metformin (solid blue triangles). All the experiments indicated in this figure were performed on the same day. These experiments were repeated 3 times and similar results were obtained.

resulted in decreased anti-apoptotic MCL1 expression. In contrast, the expression of pro-apoptotic BAX and BIM were increased upon metformin treatment. Part of the effects of metformin may be due to miR-26a expression as metformin increased its expression. miR26a mimics also decreased MCL1 expression and increased the induction of apoptosis in these cells (Wang et al., 2016).

Metformin administration may have effects on miR-155/miR-144 which is important in the regulation of mitochondrially-bound hexokinase II (mtHKII). mtHKII has important roles in the resistance of cancer cells in avoiding cell death as well as protecting against ischemia-reperfusion (IR) injury. Some of the effects of the cardioprotection against reperfusion were shown to be due to the mitochondria, such as preventing outer mitochondrial membrane breakage and subsequent cytochrome C release (Nederlof et al., 2014).

BBR is consumed by millions of people as it is contained in fruits. It is also prepared as a dietary supplement and present in capsules. As a nutraceutical, BBR is consumed for the prevention and suppression of various diseases. Various attempts by multiple investigators have been performed to increase BBR's effectiveness. In our studies, we examined the effects of modified BBRs (NAX compounds) on colony formation in four different PDAC cell lines. Some of the NAX compounds inhibited proliferation more than other NAX compounds and the parental BBR both in terms of MTT assays and $\mathrm{IC}_{50}$ determination and in colony formation abilities (Abrams et al., 2018b). Interestingly, the effects of NAX038 and NAX060 compounds were similar on all four PDAC cell lines in terms of colony formation assays. Metformin increased the ability of some, but not all, NAX compounds to inhibit proliferation.

MIA-PaCa-2 cells have a gain of function mutation at TP53 (Deer et al., 2010) and an activating mutation at KRAS. We have examined previously the effects of metformin and BBR on these cells (Abrams et al., 2018a; Abrams et al., 2018b; Candido et al., 2108; McCubrey et al., 2018). AsPC-1 and BxPC3 cells also have TP53 mutations. AsPC-1 has mutations at KRAS while BxPC3 is reported to have WT-KRAS. miR pathways would be predicted to be dysregulated in these PDAC cells as TP53 is mutated and miRs can also influence the KRAS pathway. Since TP53 and KRAS are frequently mutated in PDAC, elucidating the effectiveness of drugs and nutraceuticals which can function in the presence of these mutations is important.

The increased effectiveness of certain NAX compounds in the presence of metformin in inhibiting proliferation could be due to multiple mechanisms. Some of the more effective NAX compounds could induce more DNA damage which could synergize with the effects of metformin and result in cell death. Some of the NAX compounds could induce more ROS or WT-TP53 than others which would stimulate the induction of miRs and apoptosis. The induction of autophagy is also a possible mechanism which could explain the differences of the NAX compounds effectiveness. Elucidation of the mechanisms by which the different NAX compounds in 

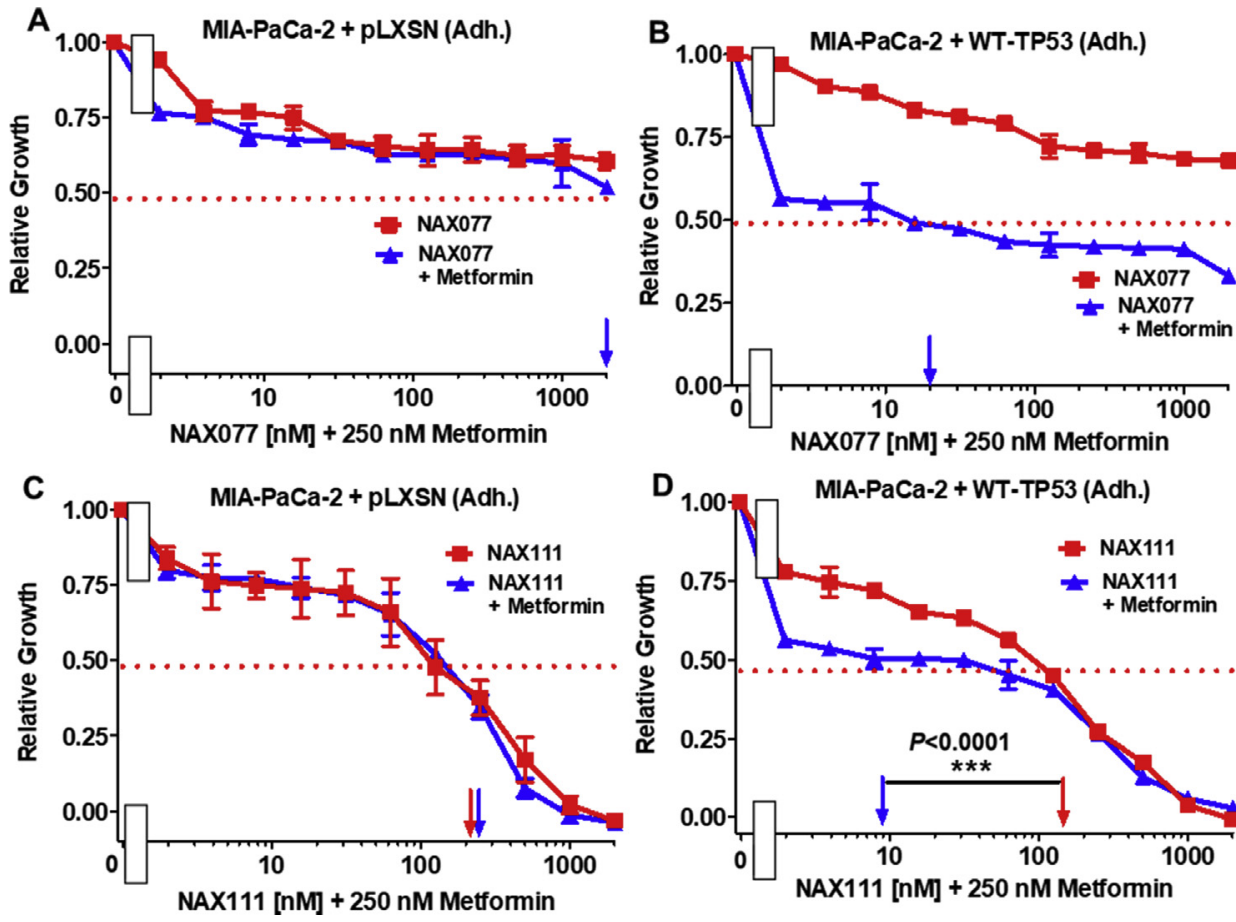

Fig. 15. Effects of the NAX077 and NAX111 Compounds on the Proliferation and IC50s of MIA-PaCa-2 + pLXSN (Adh.) and MIA-PaCa-2 + WT-TP53 (Adh.) Cells in the Absence and Presence of $250 \mathrm{nM}$ Metformin. Panel A) MTT analysis of MIA-PaCa-2 + pLXSN (Adh.) cells plated with different concentrations of NAX077 (solid red squares) or different concentrations of NAX077 and $250 \mathrm{nM}$ metformin (solid blue triangles). Panel B) MTT analysis of MIA-PaCa-2 + WT-TP53 (Adh.) cells plated with different concentrations of NAX077 (solid red squares) or different concentrations of NAX077 and $250 \mathrm{nM}$ metformin (solid blue triangles). Panel C) MTT analysis of MIA-PaCa-2 + pLXSN (Adh.) cells plated with different concentrations of NAX111 (solid red squares) or different concentrations of NAX111 and 250 nM metformin (solid blue triangles). Panel D) MTT analysis of MIA-PaCa-2 + WT-TP53 (Adh.) cells plated with different concentrations of NAX111 (solid red squares) or different concentrations of NAX111 and $250 \mathrm{nM}$ metformin (solid blue triangles). All the experiments indicated in this figure were performed on the same day. These experiments were repeated 3 times and similar results were obtained.

Table 1

Effects of Berberine and NAX Compounds on MIA-PaCa-2 + pLXSN (Adh.) Cells. ${ }^{\text {. }}$

\begin{tabular}{|c|c|c|c|c|}
\hline Compound & $\mathrm{IC}_{50}$ & $\mathrm{IC}_{50}$ with $250 \mathrm{nM}$ Metformin & Fold Difference of $\mathrm{IC}_{50}$ with $250 \mathrm{nM}$ Metformin & Statistical Significance \\
\hline Berberine (BBR) & $1600 \pm 17 \mathrm{nM}$ & $600 \mathrm{nM} \pm 5.4 \mathrm{nM}$ & $2.7 \times \downarrow$ & $P<0.0001$ \\
\hline NAX012 & $1300 \pm 2.9 \mathrm{nM}$ & $1300 \mathrm{nM} \pm 2.9 \mathrm{nM}$ & - & - \\
\hline NAX014 & $750 \pm 6.3 \mathrm{nM}$ & $750 \mathrm{nM} \pm 16 \mathrm{nM}$ & - & - \\
\hline NAX035 & $450 \pm 11.5 \mathrm{nM}$ & $8.5 \mathrm{nM} \pm 1 \mathrm{nM}$ & $52.9 \mathrm{X} \downarrow$ & $P<0.0001$ \\
\hline NAX038 & $500 \pm 12 \mathrm{nM}$ & $70 \mathrm{nM} \pm 2.8 \mathrm{nM}$ & $6.4 \mathrm{X} \downarrow$ & $P<0.0001$ \\
\hline NAX042 & $180 \pm 1.3 \mathrm{nM}$ & $150 \mathrm{nM} \pm 1 \mathrm{nM}$ & $1.2 \times \downarrow$ & $P<0.0001$ \\
\hline NAX053 & $100 \pm 1 \mathrm{nM}$ & $15 \mathrm{nM} \pm 0.4 \mathrm{nM}$ & $6.7 \times \downarrow$ & $P<0.0001$ \\
\hline NAX054 & $2000 \mathrm{nM}$ & $2000 \mathrm{nM}$ & - & - \\
\hline NAX060 & $180 \pm 12.6 \mathrm{nM}$ & $28 \mathrm{nM} \pm 2.5 \mathrm{nM}$ & $6.4 \times \downarrow$ & $P<0.0001$ \\
\hline NAX075 & $>2000 \mathrm{nM}$ & $1000 \mathrm{nM} \pm 4.9 \mathrm{nM}$ & $>2 X \downarrow$ & - \\
\hline NAX077 & $>2000 \mathrm{nM}$ & $2000 \mathrm{nM}$ & $>2 \mathrm{X} \downarrow$ & _- \\
\hline NAX111 & $200 \pm 11.3 \mathrm{nM}$ & $200 \mathrm{nM} \pm 7.8 \mathrm{nM}$ & - & - \\
\hline
\end{tabular}

a Determined by MTT Analysis as presented in Abrams et al. (2018), Candido et al. (2018); Abrams et al. (2019).

combination with metformin inhibit proliferation could further the usage of modified BBRs (NAX compounds) in cancer therapy as well as other diseases. It is conceivable that type-II diabetes patients who are treated with metformin to alleviate their diabetes also consume BBR either as a fruit or a dietary supplement. This combination could have enhanced anti-cancer effects.

In our studies, we have for the first time shown the effects of introduction of WT-TP53 on the effects of modified BBRs in response to a suboptimal dose of $250 \mathrm{nM}$ metformin. Certain NAX compounds (e.g., NAX014, NAX038, NAX042, NAX077 and NAX111) elicited elevated sensitivity in the presence of WT-TP53 and metformin while other NAX compounds (e.g. NAX053, NAX060, NAX075) showed greater effect on sensitivity to the addition of $250 \mathrm{nM}$ metformin in the absence of WT-TP53. The relationships between BBR and DNA binding and TP53 are not well established. It is possible that various NAX compounds can interact with DNA and WT-TP53 in different fashions which could be useful in certain cancer treatments. Further elucidation of the effects of NAX 
Table 2

Effects of berberine and NAX compounds on MIA-PaCa-2 + WT-TP53 (Adh.) cells. ${ }^{\text {a }}$

\begin{tabular}{|c|c|c|c|c|}
\hline Compound & $\mathrm{IC}_{50}$ & $\mathrm{IC}_{50}$ with $250 \mathrm{nM}$ Metformin & Fold Difference of $\mathrm{IC}_{50}$ with $250 \mathrm{nM}$ Metformin & Statistical Significance \\
\hline Berberine (BBR) & $900 \pm 1.9 \mathrm{nM}$ & $500 \mathrm{nM} \pm 5 \mathrm{nM}$ & $1.8 \mathrm{X} \downarrow$ & $P<0.0001$ \\
\hline NAX012 & $900 \pm 9 \mathrm{nM}$ & $550 \mathrm{nM} \pm 0.8 \mathrm{nM}$ & $1.6 \mathrm{X} \downarrow$ & $P<0.0001$ \\
\hline NAX014 & $500 \pm 2.9 \mathrm{nM}$ & $15 \mathrm{nM} \pm 0.5 \mathrm{nM}$ & $33.3 \mathrm{X} \downarrow$ & $P<0.0001$ \\
\hline NAX035 & $400 \pm 8 \mathrm{nM}$ & $300 \mathrm{nM} \pm 6 \mathrm{nM}$ & $1.3 \mathrm{X} \downarrow$ & $P<0.0001$ \\
\hline NAX038 & $220 \pm 3.2 \mathrm{nM}$ & $30 \mathrm{nM} \pm 0.4 \mathrm{nM}$ & $7.3 \times \downarrow$ & $P<0.0001$ \\
\hline NAX042 & $200 \pm 2.6 \mathrm{nM}$ & $40 \mathrm{nM} \pm 0.8 \mathrm{nM}$ & $5 X \downarrow$ & $P<0.0001$ \\
\hline NAX053 & $130 \pm 2 n M$ & $40 \mathrm{nM} \pm 0.8 \mathrm{nM}$ & $3.3 \times \downarrow$ & $P<0.0001$ \\
\hline NAX054 & $>2000 \mathrm{nM}$ & $2000 \mathrm{nM}$ & - & - \\
\hline NAX060 & $60 \pm 0.8 \mathrm{nM}$ & $40 \mathrm{nM} \pm 1.2 \mathrm{nM}$ & $1.5 \times \downarrow$ & $P<0.0001$ \\
\hline NAX075 & $>2000 \mathrm{nM}$ & $>2000 \mathrm{nM}$ & - & - \\
\hline NAX077 & $>2000 \mathrm{nM}$ & $20 \mathrm{nM}$ & $>100 \mathrm{X} \downarrow$ & - \\
\hline NAX111 & $150 \pm 2.3 \mathrm{nM}$ & $9 \mathrm{nM} \pm 0.3 \mathrm{nM}$ & $16.6 \mathrm{X} \downarrow$ & $\mathrm{P}<0.0001$ \\
\hline
\end{tabular}

a Determined by MTT Analysis as presented in Abrams et al. (2018), Candido et al. (2018); Abrams et al. (2019).

compounds and metformin in the presence and absence of WT-TP53 could further our understanding of how BBR and modified derivatives function in suppressing growth of pancreatic and other cancer cell types.

\section{Conflicts of interest}

The authors declare that they have no conflicts of interest with publication of this manuscript.

\section{Acknowledgements}

JAM, SLA, KL and LSS were supported in part by a grant from East Carolina University Grants (\#111104 and \#111110-6687150000). GR, SR and MYF were supported in part by grants from: Intesa San Paolo Foundation. DR and AG were supported in part from the Polish National Science Center grant number UMO-2015/19/B/NZ1/00332.

\section{References}

Abrams, S.L., Lertpiriyapong, K., Yang, L.V., Martelli, A.M., Cocco, L., Ratti, S., Falasca, M., Murata, R.M., Rosalen, P.L., Lombardi, P., Libra, M., Candido, S., Montalto, G., Cervello, M., Steelman, L.S., McCubrey, J.A., 2018. Introduction of WT-TP53 into pancreatic cancer cells alters sensitivity to chemotherapeutic drugs, targeted therapeutics and nutraceuticals. Adv. Biol. Regul. 69, 16-34.

Abrams, S.L., Follo, M.Y., Steelman, L.S., Lertpiriyapong, K., Cocco, L., Ratti, S., Martelli, A.M., Candido, S., Libra, M., Murata, R.M., Rosalen, P.L., Montalto, G., Cervello, M., Gizak, A., Rakus, D., Mao, W., Lombardi, P., McCubrey, J.A., 2019. Abilities of berberine and chemically modified berberines to inhibit proliferation of pancreatic cancer cells. Adv. Biol. Regul. 71, 172-182.

Adams, B.D., Kasinski, A.L., Slack, F.J., 2014. Aberrant regulation and function of microRNAs in cancer. Curr. Biol. 24, 762-776.

Andrzejewski, S., Gravel, S.,P., Pollak, M., St-Pierre, J., 2014. Metformin directly acts on mitochondria to alter cellular bioenergetics. Cancer Metabol. 2049-3002.

Arunachalam, G., Lakshmanan, A.P., Samuel, S.M., Triggle, C.R., Ding, H., 2016. Molecular interplay between microRNA-34a and Sirtuin1 in hyperglycemia-mediated impaired angiogenesis in endothelial cells: effects of metformin. J. Pharmacol. Exp. Ther. 356, 314-323.

Bao, B., Wang, Z., Ali, S., Ahmad, A., Azmi, A.S., Sarkar, S.H., Banerjee, S., Kong, D., Li, Y., Thakur, S., Sarkar, F.H., 2012. Metformin inhibits cell proliferation, migration and invasion by attenuating CSC function mediated by deregulating miRNAs in pancreatic cancer cells. Cancer Prev. Res. 5, 355-364.

Bao, B., Azmi, A.S., Ali, S., Zaiem, F., Sarkar, F.H., 2014. Metformin may function as anti-cancer agent via targeting cancer stem cells: the potential biological significance of tumor-associated miRNAs in breast and pancreatic cancers. Ann. Transl. Med. 2, 59.

Bhowmik, D., Kumar, G.S., 2016. Recent advances in nucleic acid binding aspects of berberine analogs and implications for drug design. Mini Rev. Med. Chem. 16, $104-119$.

Bhowmik, D., Das, S., Hossain, M., Haq, L., Kumar, G.S., 2012a. Biophysical characterization of the strong stabilization of the RNA triplex poly(U)·poly(A)*poly(U) by 9-O-( $\omega$-amino) alkyl ether berberine analogs. PLoS One 7, e37939.

Bhowmik, D., Hossain, M., Buzzetti, F., D'Auria, R., Lombardi, P., Kumar, G.S., 2012b. Biophysical studies on the effect of the 13 position substitution of the anticancer alkaloid berberine on its DNA binding. J. Phys. Chem. B 116, 2314-2324.

Bosetti, C., Rosato, V., Li, D., Silverman, D., Petersen, G.M., Bracci, P.M., Neale, R.E., Muscat, J., Anderson, K., Gallinger, S., Olson, S.H., Miller, A.B., Bas Bueno-deMesquita, H., Scelo, G., Janout, V., Holcatova, I., Lagiou, P., Serraino, D., Lucenteforte, E., Fabianova, E., Ghadirian, P., Baghurst, P.A., Zatonski, W., Foretova, L., Fontham, E., Bamlet, W.R., Holly, E.A., Negri, E., Hassan, M., Prizment, A., Cotterchio, M., Cleary, S., Kurtz, R.C., Maisonneuve, P., Trichopoulos, D., Polesel, J., Duell, E.J., Boffetta, P., La Vecchia, C., 2014. Diabetes, antidiabetic medications, and pancreatic cancer risk: an analysis from the International Pancreatic Cancer Case-Control Consortium. Ann. Oncol. 25, 2065-2072.

Burgeiro, A., Gajate, C., Dakir, el H., Villa-Pulgarín, J.A., Oliveira, P.J., Mollinedo, F., 2011. Involvement of mitochondrial and B-RAF/ERK signaling pathways in berberine-induced apoptosis in human melanoma cells. Anti Canccer Drugs 22, 507-518 2011.

Candido, S., Abrams, S.L., Steelman, L., Lertpiriyapong, K., Martelli, A.M., Cocco, L., Ratti, S., Follo, M.Y., Murata, R.M., Rosalen, P.L., Lombardi, P., Montalto, G., Cervello, M., Gizak, A., Rakus, D., Suh, P.G., Libra, M., McCubrey, J.A., 2018. Metformin influences drug sensitivity in pancreatic cancer cells. Adv. Biol. Regul. 68, 13-30.

Cerullo, M., Gani, F., Chen, S.Y., Canner, J., Pawlik, T.M., 2017. Metformin use is associated with improved survival in patients undergoing resection for pancreatic cancer. J. Gastrointest. Surg. 20, 1572-1580.

Chatterjee, S., Mallick, S., Buzzetti, F., Fiorillo, G., Syeda, T.M., Lombardi, P., Sahab, K.,D., Kumar, S.G., 2015. New 13-pyridinealkyl berberine analogues intercalate to DNA and induce apoptosis in HepG2 and MCF-7 cells through ROS mediated p53 dependent pathway: biophysical, biochemical and molecular modeling studies. RSC Adv. 5, 90632.

Chaiteerakij, R., Petersen, G.M., Bamlet, W.R., Chaffee, K.G., Zhen, D.B., Burch, P.A., Leof, E.R., Roberts, L.R., Oberg, A.L., 2016. Metformin use and survival of patients with pancreatic cancer: A cautionary lesson,. J Clin Oncol 34, 1898-1904.

Chen, Q., Qin, R., Fang, Y., Li, H., 2015. Berberine sensitizes human ovarian cancer cells to cisplatin through miR-93/PTEN/Akt signaling pathway. Cell. Physiol. 
Biochem. 36, 956-965.

Chen, J., Wang, T., Xu, S., Lin, A., Yao, H., Xie, W., Zhu, Z., Xu, J., 2017. Design, synthesis and biological evaluation of novel nitric oxide-donating protoberberine derivatives as antitumor agents. Eur. J. Med. Chem. 132, 173-183.

Cifarelli, V., Lashinger, L.M., Devlin, K.L., Dunlap, S.M., Huang, J., Kaaks, R., Pollak, M.N., Hursting, S.D., 2015. Metformin and rapamycin reduce pancreatic cancer growth in obese prediabetic mice by distinct microRNA-regulated mechanisms. Diabetes 64, 1632-1642.

Deer, E.L., González-Hernández, J., Coursen, J.D., Shea, J.E., Ngatia, J., Scaife, C.L., Firpo, M.A., Mulvihill, S.J., 2010. Phenotype and genotype of pancreatic cancer cell lines. Pancreas 39, 425-435.

Do, M.T., Kim, H.G., Choi, J.H., Jeong, H.G., 2014. Metformin induces microRNA-34a to downregulate the Sirt1/Pgc-1 $\alpha /$ Nrf2 pathway, leading to increased susceptibility of wild-type p53 cancer cells to oxidative stress and therapeutic agents. Free Radic. Biol. Med. 74, 21-34.

Duda, P., Wiśniewski, J., Wójtowicz, T., Wójcicka, O., Jaśkiewicz, M., Drulis-Fajdasz, D., Rakus, D., McCubrey, J.A., Gizak, A., 2018. Targeting GSK3 signaling as a potential therapy of neurodegenerative diseases and aging. Expert Opin. Ther. Targets 22, 833-848.

Esteller, M., 2011. Non-coding RNAs in human disease. Nat. Rev. Genet. 12, 861-874.

Farooqi, A.A., Qureshi, M.Z., Khalid, S., Attar, R., Martinelli, C., Sabitaliyevich, U.Y., Nurmurzayevich, S.B., Taverna, S., Poltronieri, P., Baojun, X., 2019. Regulation of cell signaling pathways by berberine in different cancers: searching for missing pieces of an incomplete jig-saw puzzle for an effective cancer therapy. Cancers 11 , E478.

Ferraroni, M., Bazzicalupi, C., Papi, F., Fiorillo, G., Guaman-Ortiz, L.M., Nocentini, A., Scovassi, A.I., Lombardi, P., Gratteri, P., 2016. Solution and solid-state analysis of binding of 13-substituted berberine analogues to human telomeric G-quadruplexes. Chem. Asian J. 11, 1107-1115.

Frazier, M.L., Fernández, E., de Llorens, R., Brown, N.M., Pathak, S., Cleary, K.R., Abbruzzese, J.L., Berry, K., Olive, M., Le Maistre, A., Evans, D.B., 1996. Pancreatic adenocarcinoma cell line, MDAPanc-28, with features of both acinar and ductal cells. Int. J. Pancreatol. 19, 31-38.

Friedman, R.C., Farh, K.K., Burge, C.B., Bartel, D.P., 2009. Most mammalian mRNAs are conserved targets of microRNAs. Genome Res. 19 , $92-105$.

Frouws, M.A., Mulde, r B.G., Bastiaannet, E., Zanders, M.M., van Herk-Sukel, M.P., de Leede, E.M., Bonsing, B.A., Mieog, J.S., Van de Velde, C.J., Liefers, G.J., 2017. No association between metformin use and survival in patients with pancreatic cancer: an observational cohort study. Medicine $96,6229$.

Gabriely, G., Wurdinger, T., Kesari, S., Esau, C.C., Burchard, J., Linsley, P.S., Krichevsky, A.,M., 2008. MicroRNA 21 promotes glioma invasion by targeting matrix metalloproteinase regulators. Mol. Cell Biol. 28, 5369-5380.

Gatto, B., Sanders, M.M., Yu, C., Wu, H.Y., Makhey, D., LaVoie, E.J., Liu, L.F., 1996. Identification of topoisomerase I as the cytotoxic target of the protoberberine alkaloid coralyne. Cancer Res. 56, 2795-2800.

Guamán Ortiz, L.M., Lombardi, P., Tillhon, M., Scovassi, A.I., 2014a. Berberine, an epiphany against cancer. Molecules 19, $12349-12367$.

Guamán Ortiz, L.M., Tillhon, M., Parks, M., Dutto, I., Prosperi, E., Savio, M., Arcamone, A.G., Buzzetti, F., Lombardi, P., Scovassi, A.I., 2014b. Multiple effects of berberine derivatives on colon cancer cells. BioMed Res. Int. 2014, 924585.

Guaman Ortiz, L.M., Croce, A.L., Aredia, F., Sapienza, S., Fiorillo, G., Syeda, T.M., Buzzetti, F., Lombardi, P., Scovassi, A.I., 2015. Effect of new berberine derivatives on colon cancer cells. Acta Biochim. Biophys. Sin. 47, 824-833.

Hirsch, H.A., Iliopoulos, D., Tsichlis, P.N., Struhl, K., 2009. Metformin selectively targets cancer stem cells, and acts together with chemotherapy to block tumor growth and prolong remission. Cancer Res. 69, 7507-7511.

Hirsch, H.A., Iliopoulos, D., Struhl, K., 2013. Metformin inhibits the inflammatory response associated with cellular transformation and cancer stem cell growth. Proc. Natl. Acad. Sci. U. S. A. 110, 972-977.

Hsu, Y.Y., Chen, C.S., Wu, S.N., Jong, Y.J., Lo, Y.C., 2012. Berberine activates Nrf2 nuclear translocation and protects against oxidative damage via a phosphatidylinositol 3-kinase/Akt-dependent mechanism in NSC34 motor neuron-like cells. Eur. J. Pharm. Sci. 46, $415-425$.

Iliopoulos, D., Hirsch, H.A., Struhl, K., 2011. Metformin decreases the dose of chemotherapy for prolonging tumor remission in mouse xenografts involving multiple cancer cell types. Cancer Res. 71, 3196-3201.

Ippolito, L., Marini, A., Cavallini, L., Morandi, A., Pietrovito, L., Pintus, G., Giannoni, E., Schrader, T., Puhr, M., Chiarugi, P., Taddei, M.L., 2016. Metabolic shift toward oxidative phosphorylation in docetaxel resistant prostate cancer cells. Oncotarget 7, 61890-61904.

Kalaiarasi, A., Anusha, C., Sankar, R., Rajasekaran, S., John Marshal, J., Muthusamy, K., Ravikumar, V., 2016. Plant isoquinoline alkaloid berberine exhibits chromatin remodeling by modulation of histone deacetylase to induce growth arrest and apoptosis in the A549 cell line. J. Agric. Food Chem. 64 , 9542-9550.

Kim, J.B., Ko, E., Han, W., Shin, I., Park, S.Y., Noh, D.Y., 2008. Berberine diminishes the side population and ABCG2 transporter expression in MCF-7 breast cancer cells. Planta Med. 74, 1693-1700.

Kou, Y., Li, L., Li, H., Tan, Y., Li, B., Wang, K., Du, B., 2016. Berberine suppressed epithelial mesenchymal transition through cross-talk regulation of PI3K/AKT and RARalpha/RARbeta in melanoma cells. Biochem. Biophys. Res. Commun. 479, 290-296.

Li, P., Jiao, J., Gao, G., Prabhakar, B.S., 2012. Control of mitochondrial activity by miRNAs. J. Cell. Biochem. 113, 1104-1110.

Li, H.L., Wu, H., Zhang, B.B., Shi, H.L., Wu, X.J., 2016. MAPK pathways are involved in the inhibitory effect of berberine hydrochloride on gastric cancer MGC 803 cell proliferation and IL-8 secretion in vitro and in vivo. Mol. Med. Rep. 14, 1430-1438.

Liu, S., Fang, Y., Shen, H., Xu, W., Li, H., 2013. Berberine sensitizes ovarian cancer cells to cisplatin through miR-21/PDCD4 axis. Acta Biochim. Biophys. Sin. 45, $756-762$.

Lonardo, E., Cioffi, M., Sancho, P., Sanchez-Ripoll, Y., Trabulo, S.M., Dorado, J., Balic, A., Hidalgo, M., Heeschen, C., 2013. Metformin targets the metabolic Achilles heel of human pancreatic cancer stem cells. PLoS One 8, e76518 2013.

Lü, Y., Han, B., Yu, H., Cui, Z., Li, Z., Wang, J., 2018. Berberine regulates the microRNA-21-ITGB4-PDCD4 axis and inhibits colon cancer viability. Oncol. Lett. 15, 5971-5976.

Luo, G., Luo, W., Sun, X., Lin, J., Wang, M., Zhang, Y., Luo, W., Zhang, Y., 2017. MicroRNA-21 promotes migration and invasion of glioma cells via activation of Sox2 and $\beta$-catenin signaling. Mol. Med. Rep. 15, 187-193.

Marverti, G., Ligabue, A., Lombardi, P., Ferrari, S., Monti, M.G., Frassineti, C., Costi, M.P., 2013. Modulation of the expression of folate cycle enzymes and polyamine metabolism by berberine in cisplatin-sensitive and -resistant human ovarian cancer cells. Int. J. Oncol. 43, 1269-1280.

McCubrey, J.A., Lertpiriyapong, K., Steelman, L.S., Abrams, S.L., Yang, L.V., Murata, R.M., Rosalen, P.L., Scalisi, A., Neri, L.M., Cocco, L., Ratti, S., Martelli, A.M., Laidler, P., Dulińska-Litewka, J., Rakus, D., Gizak, A., Lombardi, P., Nicoletti, F., Candido, S., Libra, M., Montalto, G., Cervello, M., 2017. Effects of resveratrol, curcumin, berberine and other nutraceuticals on aging, cancer development, cancer stem cells and microRNAs. Aging (N Y) 9, 1477-1536.

McCubrey, J.A., Abrams, S.L., Lertpiriyapong, K., Cocco, L., Ratti, S., Martelli, A.M., Candido, S., Libra, M., Murata, R.M., Rosalen, P.L., Lombardi, P., Montalto, G., Cervello, M., Gizak, A., Rakus, D., Steelman, L.S., 2018. Effects of berberine, curcumin, resveratrol alone and in combination with chemotherapeutic drugs and signal transduction inhibitors on cancer cells-Power of nutraceuticals. Adv. Biol. Regul. 67, 190-211.

Ming, M., Sinnett-Smith, J., Wang, J., Soares, H.P., Young, S.H., Eibl, G., Rozengurt, E., 2014. Dose-dependent AMPK-dependent and independent mechanisms of berberine and metformin inhibition of mTORC1, ERK, DNA synthesis and proliferation in pancreatic cancer cells. PLoS One 9, e114573.

Nair, V., Pathi, S., Jutooru, I., Sreevalsan, S., Basha, R., Abdelrahim, M., Samudio, I., Safe, S., 2013. Metformin inhibits pancreatic cancer cell and tumor growth and downregulates Sp transcription factors. Carcinogenesis 34, 2870-2879.

Nederlof, R., Eerbeek, O., Hollmann, M.W., Southworth, R., Zuurbier, C.,J., 2014. Targeting hexokinase II to mitochondria to modulate energy metabolism and reduce ischaemia-reperfusion injury in heart. Br. J. Pharmacol. 171, 2067-2079.

Pan, Y., Zhang, F., Zhao, Y., Shao, D., Zheng, X., Chen, Y., He, K., Li, J., Chen, L., 2017. Berberine enhances chemosensitivity and induces apoptosis through doseorchestrated AMPK signaling in breast cancer. J Cancer 8, 1679-1689.

Pierpaoli, E., Arcamone, A.G., Buzzetti, F., Lombardi, P., Salvatore, C., Provinciali, M., 2013. Antitumor effect of novel berberine derivatives in breast cancer cells. Biofactors 39, 672-679.

Pierpaoli, E., Damiani, E., Orlando, F., Lucarini, G., Bartozzi, B., Lombardi, P., Salvatore, C., Geroni, C., Donati, A., Provinciali, M., 2015. Antiangiogenic and antitumor activities of berberine derivative NAX014 compound in a transgenic murine model of HER2/neu-positive mammary carcinoma. Carcinogenesis 36, 1169-1179.

Pierpaoli, E., Fiorillo, G., Lombardi, P., Salvatore, C., Geroni, C., Piacenza, F., Provinciali, M., 2018. Antitumor activity of NAX060: a novel semisynthetic berberine 
derivative in breast cancer cells. Biofactors 44, 443-452.

Puthdee, N., Seubwai, W., Vaeteewoottacharn, K., Boonmars, T., Cha'on, U., Phoomak, C., Wongkham, S., 2017. Berberine induces cell cycle arrest in cholangiocarcinoma cell lines via Inhibition of NF-kappaB and STAT3 pathways. Biol. Pharmaceut. Bull. 40, 751-757.

Qin, Y., Pang, J.Y., Chen, W.H., Zhao, Z.Z., Liu, L., Jiang, Z.H., 2007. Inhibition of DNA topoisomerase I by natural and synthetic mono- and dimeric protoberberine alkaloids. Chem. Biodivers. 4, 481-487.

Rattan, R., Graham, R.P., Maguire, J.L., Giri, S., Shridhar, V., 2011 May. Metformin suppresses ovarian cancer growth and metastasis with enhancement of cisplatin cytotoxicity in vivo. Neoplasia 13 (5), 483-491.

Reni, M., Dugnani, E., Cereda, S., Belli, C., Balzano, G., Nicoletti, R., Liberati, D., Pasquale, V., Scavini, M., Maggiora, P., Sordi, V., Lampasona, V., Ceraulo, D., Di Terlizzi, G., Doglioni, C., Falconi, M., Piemonti, L., 2016. (Ir)relevance of metformin treatment in patients with metastatic pancreatic cancer: an open-label, randomized phase II trial. Clin. Cancer Res. 22, 1076-1085.

Sharma, P., Kumar, S., 2018. Metformin inhibits human breast cancer cell growth by promoting apoptosis via a ROS-independent pathway involving mitochondrial dysfunction: pivotal role of superoxide dismutase (SOD). Cell. Oncol. 41, 637-650.

Tillhon, M., Guamán Ortiz, L.M., Lombardi, P., Scovassi, A.I., 2012. Berberine: new perspectives for old remedies. Biochem. Pharmacol. 84, $1260-1267$.

Wang, N., Feng, Y., Zhu, M., Tsang, C.M., Man, K., Tong, Y., Tsao, S.W., 2010. Berberine induces autophagic cell death and mitoch ondrial apoptosis in liver cancer cells: the cellular mechanism. J. Cell. Biochem. 111, 1426-1436.

Wang, Y., Liu, Q., Liu, Z., Li, B., Sun, Z., Zhou, H., Zhang, X., Gong, Y., Shao, C., 2012. Berberine, a genotoxic alkaloid, induces ATM-Chk1 mediated G2 arrest in prostate cancer cells. Mutat. Res. 734, 20-29.

Wang, J., Peng, Y., Liu, Y., Yang, J., Ding, N., Tan, W., 2015. Berberine, a natural compound, suppresses Hedgehog signaling pathway activity and cancer growth. BMC Canc. 15, 595 .

Wang, F., Xu, J., Liu, H., Liu, Z., Xia, F., 2016. Metformin induces apoptosis by microRNA-26a-mediated downregulation of myeloid cell leukaemia-1 in human oral cancer cells. Mol. Med. Rep. 13, 4671-4676.

Wang, J., Yang, S., Cai, X., Dong, J., Chen, Z., Wang, R., Zhang, S., Cao, H., Lu, D., Jin, T., Nie, Y., Hao, J., Fan, D., 2016. Berberine inhibits EGFR signaling and enhances the antitumor effects of EGFR inhibitors in gastric cancer. Oncotarget 7, 76076-76086.

Wang, Y., Wu, Z., Hu, L., 2018. The regulatory effects of metformin on the [SNAIL/miR-34]:[ZEB/miR-200] system in the epithelial-mesenchymal transition(EMT) for colorectal cancer(CRC). Eur. J. Pharmacol. 834, 45-53.

Wu, C., Qin, N., Ren, H., Yang, M., Liu, S., Wang, Q., 2018. Metformin regulating miR-34a pathway to inhibit Egr1 in rat mesangial cells cultured with high glucose. Internet J. Endocrinol. 21, 6462793. https://doi.org/10.1155/2018/6462793. 2018.

Xiao, N., Chen, S., Ma, Y., Qiu, J., Tan, J.H., Ou, T.M., Gu, L.Q., Huang, Z.S., Li, D., 2012. Interaction of berberine derivative with protein POT1 affect telomere function in cancer cells. Biochem. Biophys. Res. Commun. 419, 567-572.

Xu, S., Yang, Z., Jin, P., Yang, X., Li, X., Wei, X., Wang, Y., Long, S., Zhang, T., Chen, G., Sun, C., Ma, D., Gao, Q., 2018. Metformin suppresses tumor progression by inactivating stromal fibroblasts in ovarian cancer. Mol. Canc. Therapeut. 17, 1291-1302.

You, H.Y., Xie, X.M., Zhang, W.J., Zhu, H.L., Jiang, F.Z., 2016. Berberine modulates cisplatin sensitivity of human gastric cancer cells by upregulation of miR-203. In: Vitro Cellular \& Developmental Biology-Animal. vol. 52. pp. 857-863.

Zhang, R., Qiao, H., Chen, S., Chen, X., Dou, K., Wei, L., Zhang, J., 2016. Berberine reverses lapatinib resistance of HER2-positive breast cancer cells by increasing the level of ROS. Cancer Biol. Ther. 17, 925-934.

Zhao, Y., Jing, Z., Li, Y., Mao, W., 2016. Berberine in combination with cisplatin suppresses breast cancer cell growth through induction of DNA breaks and caspase-3dependent apoptosis. Oncol. Rep. 36, 567-572. 